\title{
High Resolution Imaging of Vadose Zone Transport Using Surface and Crosswell Ground Penetrating Radar Methods
}

November 2002

Kenneth H. Williams, Mike B. Kowalsky, and John E. Peterson

Lawrence Berkeley National Laboratory 


\section{Introduction}

To effectively clean up many contaminated sites there is a need for information on heterogeneities at scales ranging from one centimeter to tens of meters, as these features can alter contaminant transport significantly. At the Department of Energy's Hanford, Washington site heterogeneities of interest can range from localized phenomena such as silt or gravel lenses, fractures, clastic dikes, to large-scale lithologic discontinuities. In the vadose zone it is critical to understand the parameters controlling flow. These features have been suspected of leading to funneling and fingering, additional physical mechanisms that could alter and possibly accelerate the transport of contaminants to underlying groundwater. For example, it has been observed from the studies to date that over relatively short distances there are heterogeneities in the physical structure of the porous medium and structural differences between repacked soil cores and the field site from which the materials initially came (Raymond and Shdo, 1966). Analysis of cores taken from the vadose zone (i.e., soil surface to water table) has been useful in identifying localized zones of contamination. Unfortunately, these analyses are sparse (limited to a few boreholes) and extremely expensive. The high levels of radioactivity at many of the contaminated sites increase drilling and sample costs and analysis time. Cost of drilling and core analysis for the SX tank farm has exceeded \$1M per borehole (50 meter deep) for sampling. The inability to track highly mobile species through the vadose zone highlights an important need: the need for methods to describe the complete vadose zone plume and to determine processes controlling accelerated contamination of groundwater at Hanford. A combination of surface and crosswell (i.e. borehole) geophysical measurements is one means to provide this information.

The main questions addressed with the radar methods in this study are:

- What parts of the vadose zone-groundwater system control flow geometry?

- What physical properties or mechanisms control flow and transport in unconsolidated soils of the vadose zone?

- What is the optimum suite of field tests to provide information for predicting flow and transport behavior? 
- How can the information obtained during site characterization be used for building confidence in predictive numerical models?

Fully developed, application of geophysics should enable location of contaminant distributions. Questions addressed in this study were the sensitivity, resolution, and accuracy of the geophysical methods in order to derive the spatial and temporal distribution of properties controlling transport and contaminant distribution between and away from boreholes and the surface. Implicit in this activity is that geophysical methods will be used to extrapolate and extend measurements made at the point scale to the volumetric scale.

Overall there are two broad hypotheses being addressed in the geophysical work:

(1) Geophysical methods can identify physical and chemical heterogeneity controlling contaminant transport at a meaningful scale.

(2) Geophysical methods have the sensitivity to directly or indirectly detect the location of introduced fluids and/or contaminants at relevant concentrations (i.e. the subsurface has been altered from its natural state enough to create anomalies that can be detected in terms of a combination of mechanical, electrical, and thermal effects).

Examples of specific applications are:

(1) Change in moisture content in the vadose zone

(2) Location and distribution of heterogeneities within the lithology (sills, clastic dikes, fractures/faults, fracture orientation/density etc.)

(3) Matrix and fracture properties of units (porosity, grain size distribution, moisture distribution, mineral composition, etc.).

(4) Location of "fast paths" affecting transport (natural and contaminant induced) 


\section{Approach}

The twin purposes of the surface and crosswell (i.e. borehole) radar imaging were to provide information on the lithology and structure at the clastic dike vadose zone (CDVZ) transport field site and to provide some detail on the location of fluid transport during a carefully controlled injection experiment. The focus of the measurements was to determine the state variables controlling water movement (e.g. water content, physical heterogeneities and if possible water potential and chemical concentrations). This was accomplished by using time lapse radar measurements to measure changes in the dielectric constant of the subsurface.

There was a principal injection event at the CDVZ site that was monitored repeatedly with both the surface and crosswell radar methods. The water was introduced as part of a 60-meter drip line injection that spanned a period of roughly three months. The water was introduced in such a way that steady state was reached and further infiltration occurred at roughly a continuous flow rate. In addition to the methods of geophysical data acquisition, a variety of other measurements were made along the drip line in order to constrain changes in moisture content, matric potential, and tracer migration.

Central to the investigation was the influence of a well-exposed clastic dike that crossed the drip line approximately 50-meters from the northwest starting point of the line (Figure 1). The surface radar measurements were confined to the roughly 60 -meter portion of the injection line that crossed over the clastic dike. Conversely, the access boreholes used for the crosswell radar data acquisition allowed those regions both vertically and horizontally distant from the injection source in the area of the clastic dike to be investigated. Although moisture migration was not presumed to be preferentially horizontal in nature, the results presented below suggest that in the region of the dike, this may in fact be the case. 


\section{Methodology}

\section{Surface Radar Data}

The CDVZ test site was surveyed using a Sensors and Software, Inc. PulseEKKO1000 ground penetrating radar (GPR) system equipped with both $450 \mathrm{MHz}$ and $225 \mathrm{MHz}$ antennas. Previous GPR work at this site indicated that these two frequencies would be most appropriate for sampling the upper 2- to 3-meters of the sedimentary sequence. Surveys were completed using both common offset (i.e. fixed antenna separation) and common mid-point (CMP) techniques both prior to the infiltration and seven times after initiation of the injection. The dates of data acquisition are listed in Table 1.

\begin{tabular}{ccc}
\hline Date & Surface Radar Acquisition & Crosswell Radar Acquisition \\
\hline \hline $5 / 10 / 02$ & $\mathrm{x}$ & $\mathrm{x}$ \\
$5 / 21 / 02$ & & \\
$6 / 3 / 02$ & $\mathrm{x}$ & $\mathrm{x}$ \\
$6 / 6 / 02$ & $\mathrm{x}$ & $\mathrm{x}$ \\
$6 / 13 / 02$ & $\mathrm{x}$ & \\
$6 / 24 / 02$ & $\mathrm{x}$ & $\mathrm{x}$ \\
$7 / 29 / 02$ & $\mathrm{x}$ & \\
$8 / 12 / 02$ & $\mathrm{x}$ & \\
$9 / 4 / 02$ & & \\
\hline
\end{tabular}

Table 1: Surface and crosswell acquisition dates (injection started 05/31/02).

The common offset technique is the principal survey methodology used in interpreting the surface radar data. It forms the basis for identifying discreet reflectors at a variety of depths in the sedimentary sequence, and it is the technique used to map the reflectors both spatially and temporally during the course of the experiment. The technique is based on measuring the velocity of the sediment overlying a given reflector, which then allows estimation of the average water content throughout this sediment. The common offset technique allows the estimate of water content to be made much more rapidly than with the CMP technique. The method works as follows: 
(1) A given reflector is identified in the "baseline" common offset data.

(2) The bulk velocity of the sedimentary sequence overlying this reflector is determined by evaluating the CMP data acquired nearest to this location. Due to the fact that data from only two CMP locations was acquired, reflector velocities were determined using only a small subset of the overall line length. Given the mostly uniform initial conditions of the experiment, this was deemed a reasonable approach.

(3) The velocity estimation then allows the actual depth to the reflector to be determined using the two-way travel time to the reflector (which is the actual value recorded by the radar equipment).

(4) Utilizing the calculated "baseline" reflector depth, the subsequent electromagnetic (i.e. radar) velocities can be calculated for each acquisition visit.

(5) These velocities $(v)$ may then be converted to the real part of the dielectric constant, ( $\square$ ), by way of the following relationship: $\square=(c / v)^{2}$; where $c$ is the plane-wave propagation velocity of electromagnetic waves in free space.

(6) Either a generalized or a site-specific petrophysical relationship may then be used to convert $\square$ to volumetric water content $\left(\square_{\mathrm{v}}\right)$. In the case of this investigation, the generalized relationship determined by Topp (1980) was utilized where $\square_{v}=0.053+$ $0.0292 \square+0.00055 \square^{2}+0.0000043 \square^{3}$.

The common offset profiles were collected parallel to the drip line and within the shallow trench excavated for this line. Two lines were collected for each frequency (Profile\#1 and \#2) with the following parameters: $450 \mathrm{MHz}$ data acquired every $5-\mathrm{cm}$ and $225 \mathrm{MHz}$ data acquired every $10-\mathrm{cm}$. Profile\#1 consisted of a transect parallel to the drip line with a distance of $80-\mathrm{cm}$ from the center of the antenna to the nearest metal time domain reflectometry (TDR) probe. Profile\#2 consisted of a transect parallel to the drip line with a distance of $30 \mathrm{~cm}$ from the center of the antenna to the nearest metal TDR probe. Both profiles as subsequently referenced in this report with the starting position (i.e. $0-\mathrm{m}$ ) occurring at the most northwestern end of the drip line. The layout and arrangement of the profiles relative to the drip line are shown in Figure 1 and a typical common offset GPR dataset is presented in Figure 2. 


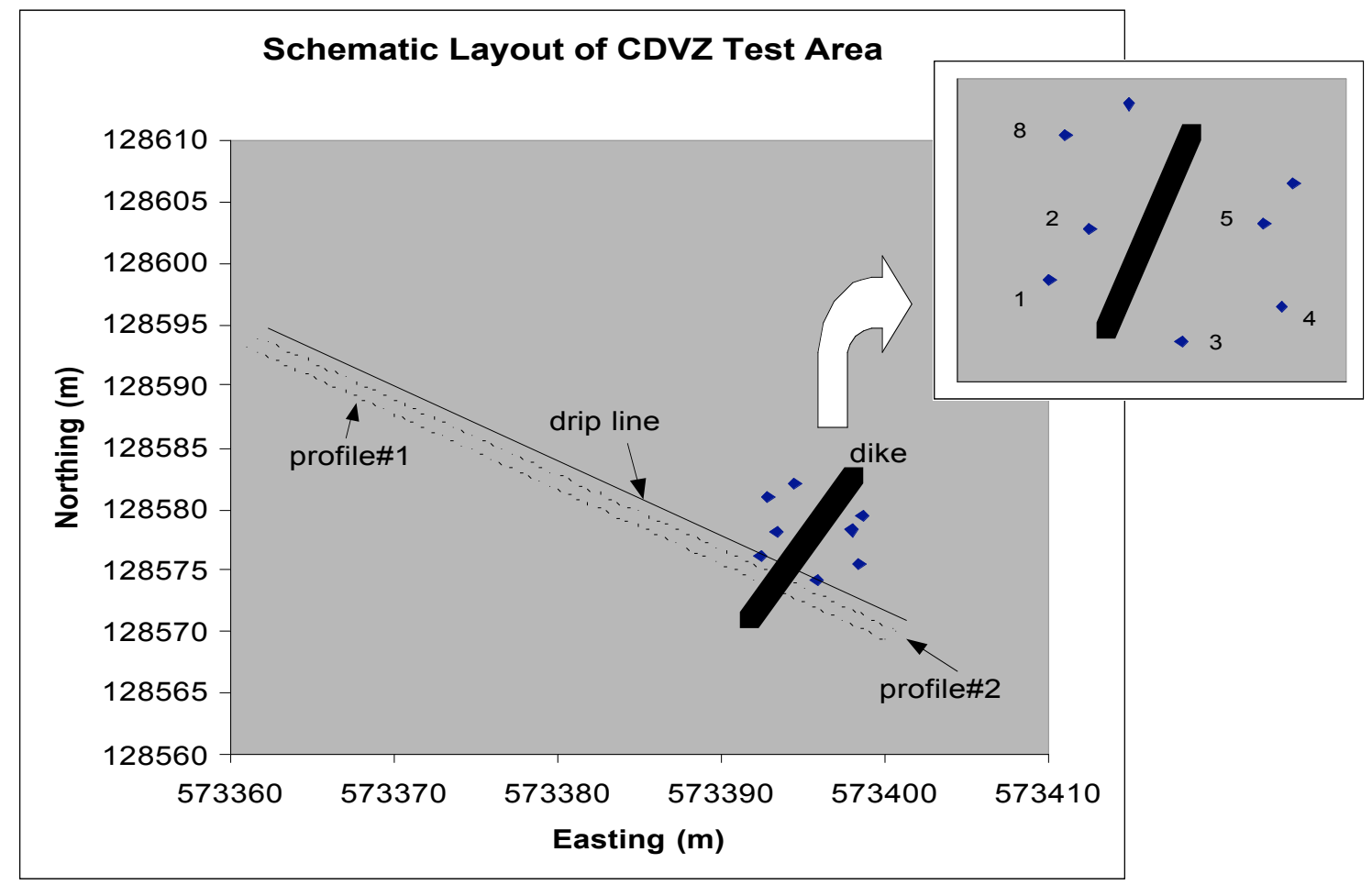

Figure 1: Schematic layout showing common offset profiles and borehole radar locations.

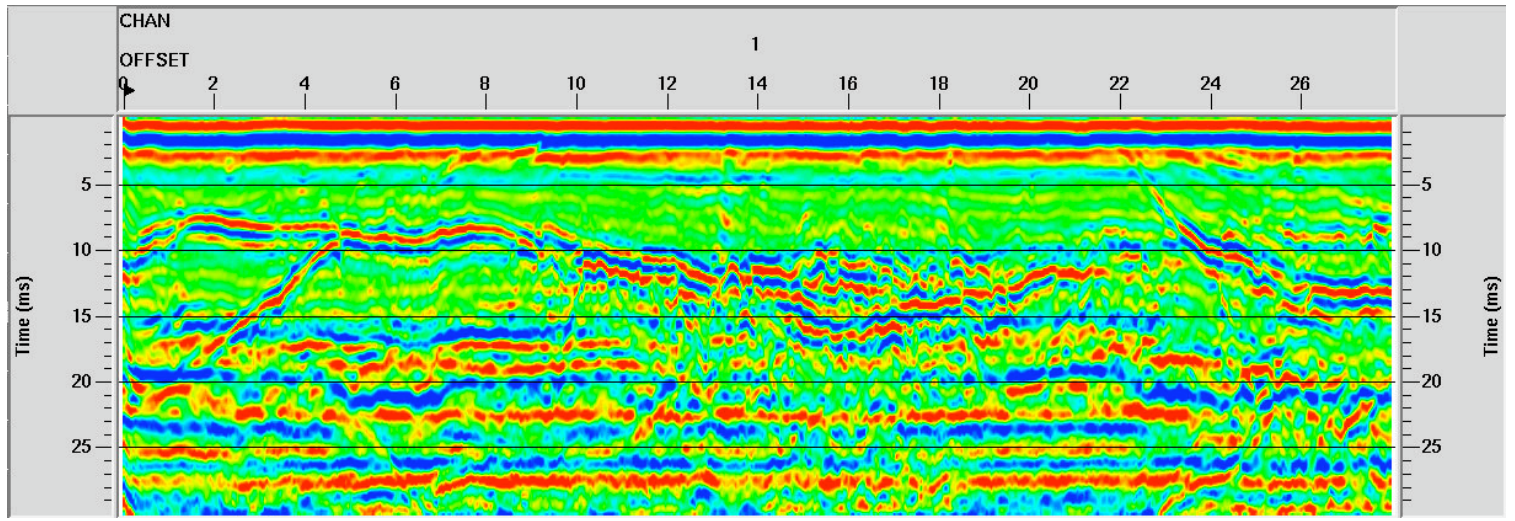

Figure 2: Common offset GPR data collected along Profile\#1 - Baseline (05/21/02)

The CMP data were collected at two specific locations along the profiles during each acquisition visit. One was located directly above the clastic dike ( $\sim 50$-meters from the starting point) and the other at about 40 -meters from the starting point. As the name 
implies, the CMP method involves the iterative movement of the radar antennas away from a common origin. The $450 \mathrm{MHz}$ antennas started with an antenna separation of 26$\mathrm{cm}$ and were moved away from one another in steps of 2-cm (i.e. the transmitting antenna and receiving antenna each moved $1-\mathrm{cm}$ ). The $225 \mathrm{MHz}$ antennas started with an antenna separation of 26-cm and they were moved away from one another in steps of 4-cm. The characteristic features of the CMP survey include the air and direct ground wave arrivals as well as any reflections that occur. The move-out (i.e. wavelet slope) of each of these arrivals defines the velocity of the arrival, or in the case of a reflector, the average velocity of the overlying sediments. A typical CMP dataset is presented in Figure 3. It should be noted that the units of velocity in the figure are incorrectly labeled as " $\mathrm{ft} / \mathrm{s}$ " due to a processing package error; the correct units are "mm/ns" (i.e. $290-\mathrm{ft} / \mathrm{sec}=290-\mathrm{mm} / \mathrm{ns}$ or $0.29-\mathrm{m} / \mathrm{ns}$ ). Further, the units of time along the vertical axis should read nanoseconds (ns) rather than milliseconds (ms).

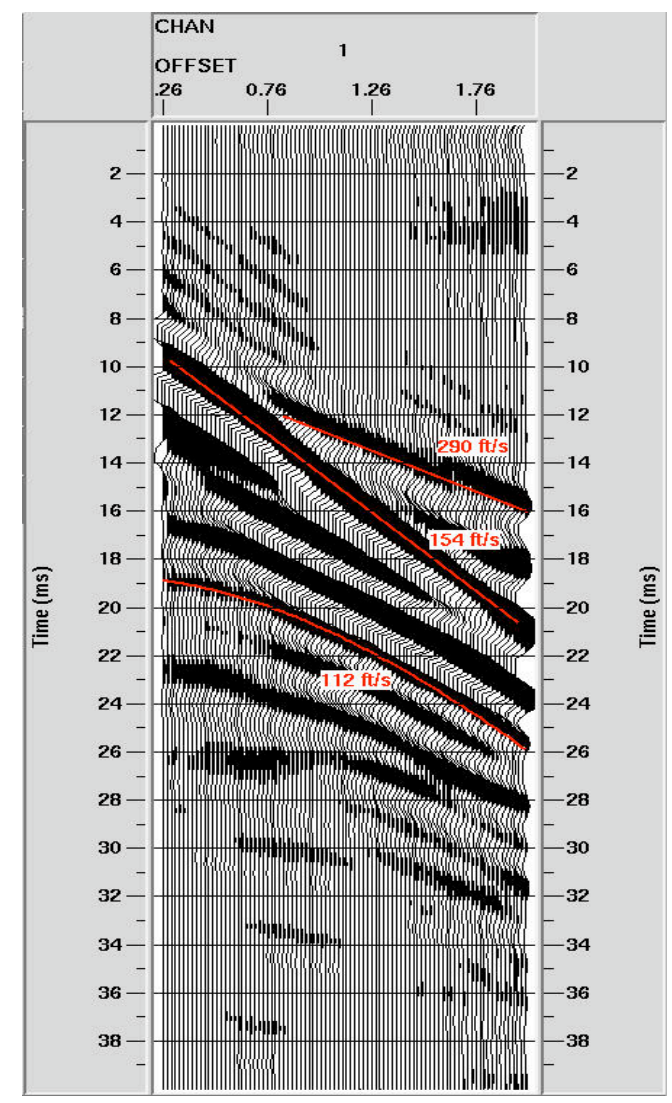

Figure 3: Common mid-point data at position E140 with arrivals labeled. 
As mentioned above, the velocity of a given reflector determined using the CMP data is then used to determine the depth to a given reflector using the recorded two-way travel time from transmitter to reflector to receiver. Given sufficient CMP locations along the length of the common offset profile, the velocity as a function of depth for a variety of reflectors can be assessed. Unfortunately, the CMP locations chosen for this investigation yielded only one or two usable reflectors, and these occurred only for the location 40-m from the profile starting point. As can be seen in Figure 3, the most prominent reflector occurs at roughly $19-\mathrm{ns}$ and has a velocity of $0.112-\mathrm{m} / \mathrm{ns}$. The other two reflectors are marked with their velocities and represent, from top to bottom, the direct airwave arrival $(0.290-\mathrm{m} / \mathrm{ns} \square 0.3-\mathrm{m} / \mathrm{ns})$ and the ground wave arrival $(0.154-\mathrm{m} / \mathrm{ns})$.

\section{Borehole Radar Data}

The borehole radar data acquired at the CDVZ test site was surveyed using a Sensors and Software, Inc. PulseEKKO100 ground penetrating radar (GPR) system equipped with both $100 \mathrm{MHz}$ and $200 \mathrm{MHz}$ antennas. The $100 \mathrm{MHz}$ antennas were used for those well pairs in which the signals traversed the clastic dike (well pairs 1-3 and 4-8) while the $200 \mathrm{MHz}$ antennas were used for all the remaining well pairs (well pairs 1-2, 2-8, 3-4, and 4-5). The reason for the lower frequency requirement for those pairs crossing the dike was likely due to its higher background moisture content and possibly some remnant of sulfate salts that remained sequestered from an infiltration experiment the previous year. This possibility is explored in more detail in the results sections below. The borehole radar technique utilized during the CDVZ transport test was a borehole radar profiling method in which the transmitter and receiver antennas were located in separate boreholes and data were collected with the antennas at various vertical offsets. The locations and naming convention for the boreholes is shown in the expanded view in Figure 1. The data collection was performed using two acquisition modes. The first was a Zero Offset Profile (ZOP) in which the transmitter and receiver antennas were positioned within the boreholes at equal depths such that there was no vertical offset. The second was a Multiple Offset Profile (MOP) in which the receiving antenna remained at a fixed depth while the transmitter antenna was moved incrementally in the second borehole. Each 
multiple offset profile constitutes a receiver gather and the compilation of these gathers is then used to construct tomographic images.

Over the course of the experiment, the radar system was operated using identical acquisition parameters for each of the four field surveys: one occurred before the injection was initiated and three followed at various intervals over the course of the study (see Table 1 for specific dates). No adjustments, filters or gains are applied to the stored raw data. Therefore, data acquisition and hence data repeatability is the same regardless of who operates the system and when - so long as the antenna configuration is the same. Data repeatability is absolutely tantamount to successful tomographic differencing and interpretation. Small deviations in experimental methodology at such close borehole spacing can result in large discrepancies in data processing.

The most important information to be obtained from radar data is the travel times, which are inverted for the velocity structure between boreholes. It is important to know the precise time when the transmitter fires (known as time-zero), to determine accurate travel times between the transmitter and receiver antennas. Direct airwave measurements (the signal from transmitter antenna to receiver antenna in air) with the antennas held at a separation of $2.0-\mathrm{m}$ were taken to help determine the zero-time. After these measurements were taken, the antennas were immediately moved into the boreholes and a ZOP dataset was collected, concluding with another set of measurements in air at the borehole collars and together in air. Following this procedure, the MOP datasets were then collected with the locations determined before the start of the survey. In the case of the CDVZ surveys, the transmitter and receiver intervals were every 0.125 meters. As in all MOP gathers, the receiver antenna remained at a fixed location (1-m, 1.125-m, 1.25$\mathrm{m}$, etc.) while the transmitter antenna occupied each of its possible locations up the borehole (e.g., $6.25-\mathrm{m}$ to $1.0-\mathrm{m}$ at $0.125-\mathrm{m}$ spacing). Each of the necessary waveforms was collected and recorded for the subsequent tomographic processing. Following MOP 
acquisition, a final ZOP dataset is collected as described above. This is done in an attempt to estimate the time-zero drift that unavoidably occurs.

\section{Determining the zero time and estimation of drift}

The zero time is defined as that instant the source emits a signal. The determination of this time is essential for the inversion of travel times for velocity and any differencing of times between data sets. The zero time was determined with the antennas held in a medium with a known velocity (i.e. $\mathrm{v}_{\text {air }}=0.30-\mathrm{m} / \mathrm{ns}$ ) at a fixed distance $(2.0-\mathrm{m})$. By determining the travel time for this separation, the zero-time may be directly calculated. This value was subtracted from the ZOP data to find the absolute time for this data. An equivalent ZOP profile was extracted from the MOP data set that could be compared to the ZOP profile acquired from the field. The zero time was subtracted from this pseudoZOP profile and if the travel times match, then this is taken to be the zero time. When the times were offset, the average offset time was calculated and the MOP zero times were corrected for this value. This proved to provide an accurate measure of zero time throughout the surveys and a way to account for any drift that may have occurred.

\section{Processing and inversion}

The travel times resulting from the aforementioned waveforms were picked and used to perform a velocity inversion for each of the four surveys. These travel times were picked using processing software to display the picks. Any error in picking is dependent primarily on the zero time adjustment and the repeatability of transmitter/receiver locations. These are both quite accurate and produce a picking error of less than one sample or $0.4 \mathrm{~ns}$. The picks are output into a file that can be used for the inversion program. Travel time tomography uses the first arrival times picked from the recorded MOP waveforms for each transmitter-receiver pair to obtain an estimate of the twodimensional inter-borehole velocity structure based on an inversion algorithm. The 
inversion method is based on the relation between propagation velocity and the total travel time along a raypath between thee transmitter and receiver:

$$
T=\prod_{B} u(\boldsymbol{r}) d l
$$

where $T$ is the travel time, $u(\boldsymbol{r})$ is the slowness, or inverse velocity, at a coordinate in space $\boldsymbol{r}$ and $d l$ is the incremental distance along the raypath with total length $R$. When inverting the travel times for slowness, the region of interest is generally divided into cells of constant slowness. Under these discretized conditions, Eq. 1 can be described by a set of linear equations

$$
t_{k}=\square_{i=1}^{N} u_{i} l_{k i}
$$

where $t_{k}$ is the travel time of the $k$ th raypath, $u_{i}$ is the slowness estimate of the $i$ th cell, $l_{k i}$ is the length of the $k$ th raypath in the $i$ th cell, and $N$ is the total number of cells. An Algebraic Reconstruction Technique (CART V1.0), as described in Peterson et al. (1986), is used in the travel time inversion for velocities with the resulting image constituting a velocity tomogram with $N$ number of cells.

Subtracting the velocity values between any two tomograms can highlight the differences in velocity from one acquisition visit to the next. Furthermore, both the absolute velocity values and the differenced (i.e. subtracted) velocity values may then be converted to their corresponding values of $\square$. The values of $\square$ may then be used to determine the $\square_{\mathrm{v}}$ values for each cell in the tomogram using Topp's equation as described above. The resulting tomograms may then be compared to the appropriate neutron probe data in order to constrain the estimates of $\square_{\mathrm{v}}$. In a similar fashion, the recorded radar waveforms may also be evaluated in terms of their wave amplitudes (here defined as the maximum peak to peak amplitude), and this information may then inverted for corresponding attenuation coefficient as follows: 


$$
\square=\square\left(\ln A / A_{o}\right) / x
$$

where $\square$ is the attenuation coefficient in $\mathrm{dB} / \mathrm{m}, \mathrm{A}$ and $\mathrm{A}_{\mathrm{o}}$ are the amplitudes of the postinfiltration and the baseline radar waveforms, respectively, and $x$ is the distance the radar wave traveled through the medium. Whereas the velocity information provides a direct means of estimating changes in moisture content, any changes in the attenuation coefficient over time most likely represent the combined effects of both increasing moister content and pore fluid conductivity. In certain instances where the composition of the injected fluid is sufficiently conductive, this can provide a very powerful means of tracking the fluid when the background moisture content of the sediments is initially very high. Under such conditions, any change in velocity due to a marginal increase in volumetric moisture content (e.g. $\square_{\mathrm{v}}=0.31$ to $\square_{\mathrm{v}}=0.33$ ) will be sufficiently small to provide only minimal contrast over time. Conversely, the attenuation coefficient over time will change dramatically should the fluid be of contrasting electrical conductivity.

\section{Data and Results}

\section{Surface Radar Data}

As described in the methodology section above, the two types of surface GPR data processed during this investigation were common offset and common mid-point data. Upon review of the common offset GPR data, it became evident that the $225 \mathrm{MHz}$ data was of poor enough quality that its usefulness over the three-month investigation would be limited at best. Due to its lower frequency content, the $225 \mathrm{MHz}$ data was initially hoped to provide some information on that portion of the sediment sequence deeper than $\sim 1.5$ meters. Unfortunately, the signal-to-noise ratio of the $225 \mathrm{MHz}$ data was particularly poor in this depth range (especially as the injection progressed) and the resolution in the upper 1.0-meter was worse in comparison to the $450 \mathrm{MHz}$ data. As such, only the $450 \mathrm{MHz}$ data were used for this investigation. Excellent data quality over the 
duration of the experiment ensured that a variety of reflectors, spanning a range of depths from $0.20-\mathrm{m}$ to $1.20-\mathrm{m}$, could consistently be picked from one visit to the next. As shown in Figure 2, a series of strong reflectors exist in the baseline data occurring at times ranging from 10 to 20 -ns. These reflectors were shown to be present over the duration of the experiment with the only change being a gradual decrease in the signalto-noise ratio as the total fluid volume injected increased. Given the confidence that a consistent series of reflectors could be identified over the duration of the experiment, a total of eight reflectors were designated as follows: red1, red3, red4, red5a, red7, red8, red9, and blue2. These eight reflectors formed the basis for the subsequent moisture content calculations.

\section{Raw Data Processing Sequence}

Before proceeding directly to the results, it is worthwhile to consider the flow of the data from the raw state to the final, processed state. Kevin Bergstrom of Bechtel Hanford, Inc. periodically provided the GPR data with the data acquisition dates as listed in Table 1. These data were then converted from their native format to an ascii format so that they could be input into a commercial signal-processing package (Promax). Within this software package, the data could be manipulated through the application of a variety of filter and gain settings to maximize the resolution of those features (i.e. reflectors) of interest. The filter and gain settings were as follows: band pass filtering with corners of $150-200 \mathrm{MHz}$ and $900-1400 \mathrm{MHz}$ and an automatic gain control (AGC) setting of 10 . Additionally, the gained and filtered data were velocity migrated such that any strongly dipping reflectors present along the profile were remapped to their spatially correct positions. Due to the nature of reflection-based survey techniques, this last step is particularly important as the true spatial location of a reflector may be incorrectly represented if not properly migrated. The velocity range over which the migration was performed was determined by the nearest CMP data location (see below) and was held constant for the 25 -ns two-way travel time encompassed by the reflection surveys. This velocity value was fixed at $0.100-\mathrm{m} / \mathrm{ns}$. 
Before the reflectors were picked according to their travel times and converted to depth, the records were flattened such that the direct airwave arrival from transmitter to receiver was positioned at time zero. By making use of the CMP data file, this value was determined for each of the seven surveys. As shown in Figure 3, those surveys performed early in the CDVZ experiment when the ground was especially dry (i.e. of high velocity) illustrate the problem of air-wave/ground-wave overlay. In these instances, the higher frequency airwave arrival must be extrapolated as to its arrival time at the closest transmitter-receiver offset $(0.26-\mathrm{m})$. In the case of Figure 3, this arrival time can be seen to be $\sim 9.2-n s$. This time (9.2-ns) is then used to crosscheck the reflection profiles to verify that the first energy arrivals in the record correspond to the airwave. As denoted by the term, this arrival represents the effective zero-time at which the transmitter was fired - minus the travel time of an electromagnetic wave in air at a separation of $0.26-\mathrm{m}(0.86-\mathrm{ns})$. The reflection profiles were then "corrected" such that the starting time of each profile was that of the airwave arrival, or more correctly the initial firing time of the transmitter. This is illustrated in Figure 2 as the bold red-bluered reflector at the top (i.e. zero-time position) of the profile. All of the travel times to any of the aforementioned eight reflectors were then referenced to the true time-zero of the system.

\section{Reflector Mapping and Depth, Dielectric, and Volumetric Water Content Determination}

Once the profiles were adjusted such that time-zero (i.e. 0-ns) occurred at the start of each record, the travel times of the eight reflectors could be determined. This was accomplished using the Promax software package, which allows the reflector to be picked, and the corresponding travel times to be written to an output file. As the depth of the eight identified reflectors was not known a priori, an initial estimate of velocity was made for the baseline data using the one relevant CMP dataset. From this dataset, it was determined that the velocity value for the upper one-meter of sediment was $0.112-\mathrm{m} / \mathrm{ns}$. Using this value and the travel time to each reflector, both the depth to each of the eight reflectors and the dielectric constant $(\square)$ of the overlying sediment were determined according to the following relationships: 


$$
\begin{gathered}
d=(t \square v) / 2 \\
\square=[c \square t / 2 \square d]^{2}
\end{gathered}
$$

where $d$ is the depth (m) to the reflector, $t$ is the two-way travel time (ns) to the reflector, $v$ is the velocity of the formation $(\mathrm{m} / \mathrm{ns})$ prior to infiltration, and $c$ is the velocity of electromagnetic waves in free space $(0.3-\mathrm{m} / \mathrm{ns})$.

For the duration of the investigation, these depths and values of $\square$ became the reference points for estimating the change in moisture content for all of the subsequent GPR surveys. Given these values, the initial value of $\square_{v}$ was determined along the length of the profile using the equation stated above in the surface GPR approach section. Unfortunately, the limited CMP data available along the length of the profiles and the lack of any TDR data at the time of the data processing meant that a uniform velocity was used. This meant that the baseline $\square_{v}$ was identical along the length of the profile with the value determined to be 0.129 . Recalling that this value is an average for the upper 1.0-m of sediment, it is actually in reasonably close agreement with similarly averaged data acquired via the neutron probe logging. This data will be discussed in more detail in the subsequent section on the borehole radar data. It should be emphasized that upon availability and incorporation of the TDR probe data, a more realistic baseline moisture content profile can be determined. As will be shown in the results below, introducing greater variability in the baseline moisture content would bring the ultimate changes in $\square_{v}$ into better agreement with the expectations of the experiment.

With the above baseline data in hand, all of the subsequent GPR datasets were evaluated in terms of their reflector travel times and the corresponding change in both dielectric constant and volumetric water content. The sequence of data processing is schematically illustrated in Figure 4 with the flow being as follows: raw data input and reflector picking, depth determination, and calculation of $\square_{v}$. 

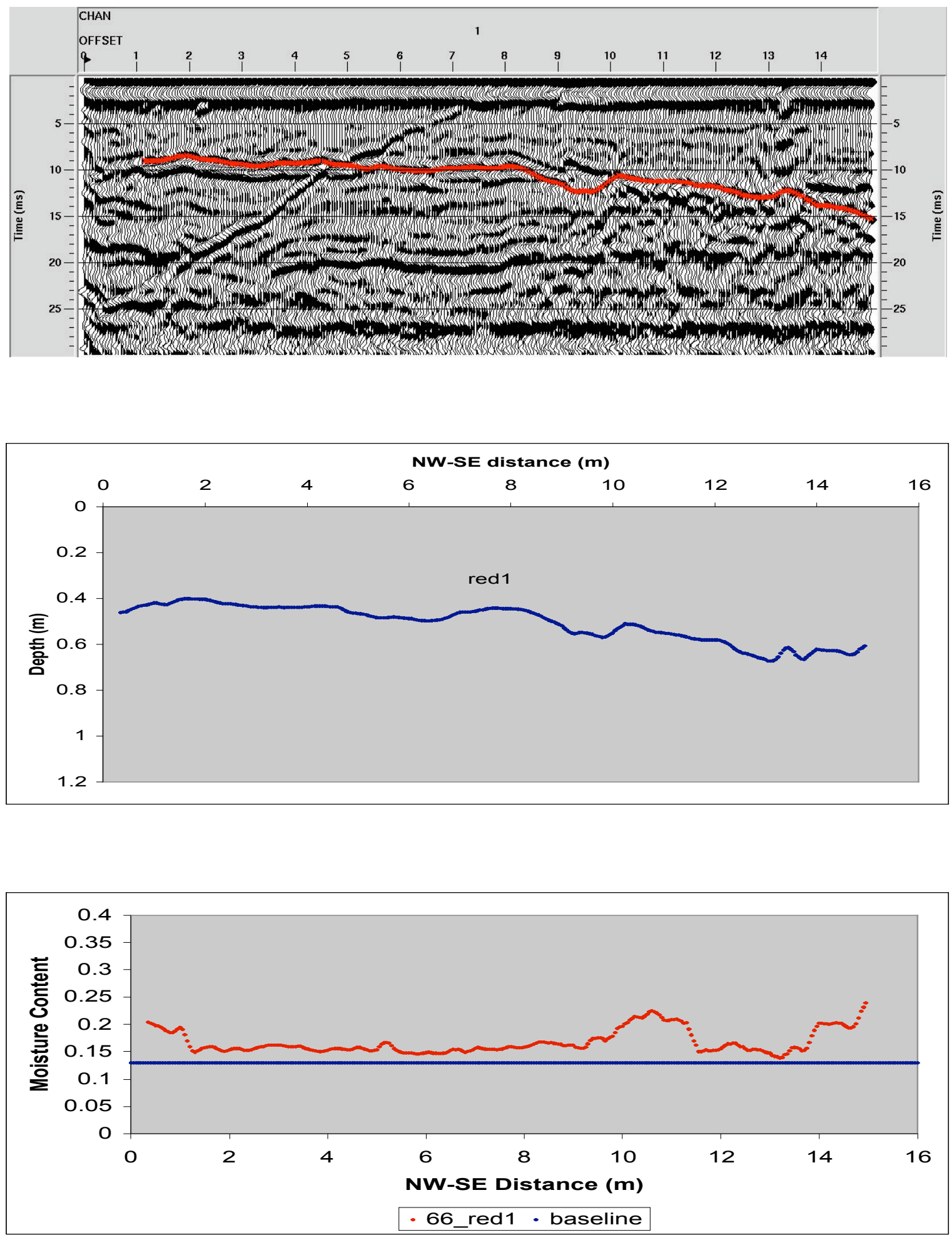

Figure 4: Schematic illustration of surface GPR processing sequence. 


\section{Surface Radar Results}

Using the aforementioned approach, the depth to each of the eight reflectors was determined with the results shown in Figure 5. As can be seen, the depths covered by the survey range from roughly $0.25-\mathrm{m}$ to $1.20-\mathrm{m}$ below ground surface. The fact that such a range of depths was represented allowed the downwardly migrating moisture front to be tracked as the infiltration progressed. The resulting time-lapse changes in travel time were monitored with the corresponding changes in $\square_{v}$ computed and displayed in Figures 6-13. Each of the figures shows the $\square_{v}$ values calculated for an individual reflector for a given acquisition date. The notation used in the legend for each plot refers to the date of acquisition and the reflector under consideration (e.g. 66_red1 represents the $\square_{v}$ calculated for reflector 'red1' on 06/06/02). Recall that the baseline value of $\square_{v}$ was 0.129 and that it was assumed to be constant along the entire length of the profile. For this reason, it is only included on the first of the eight graphs.

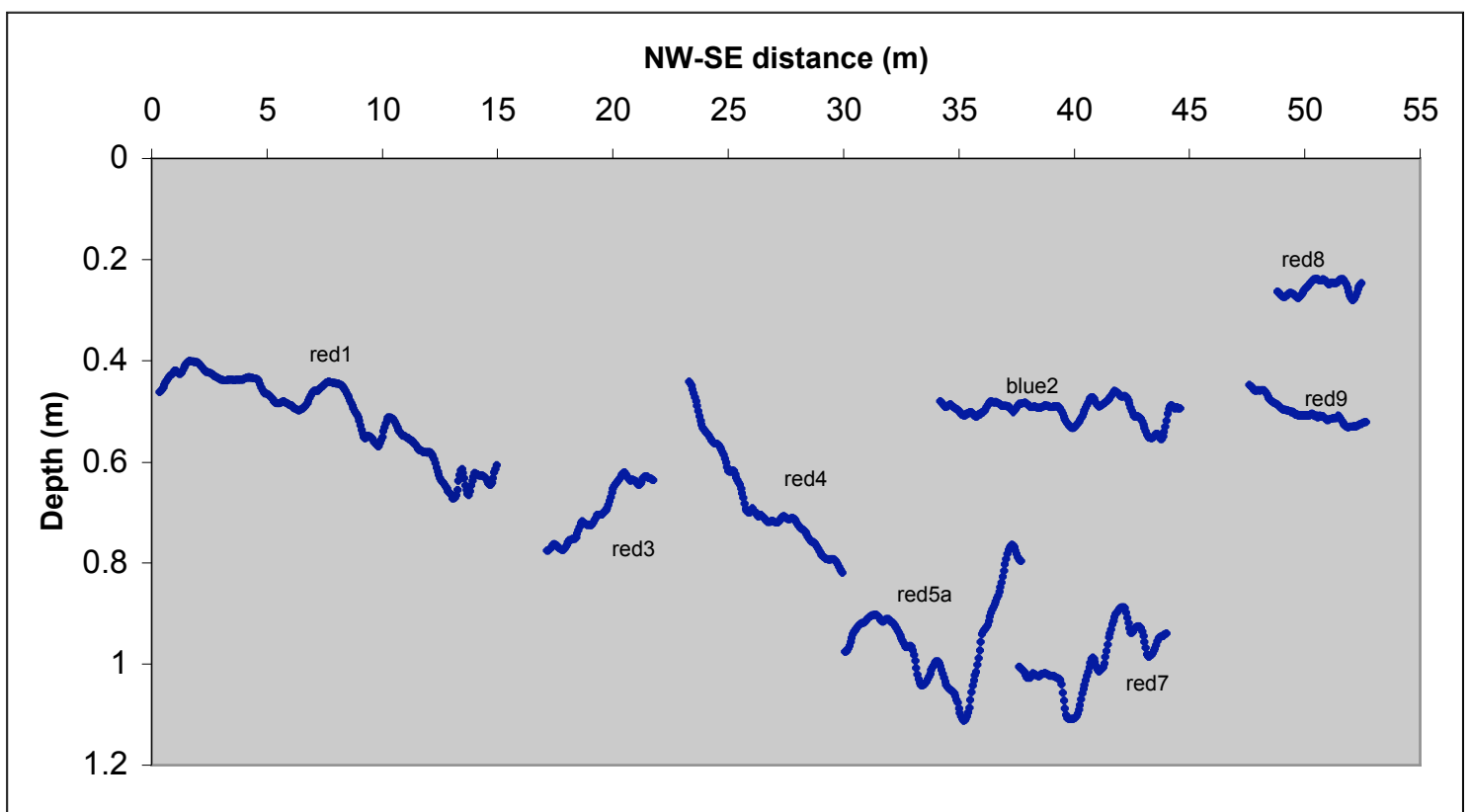

Figure 5: Map showing the spatial location of each of the eight reflectors. 


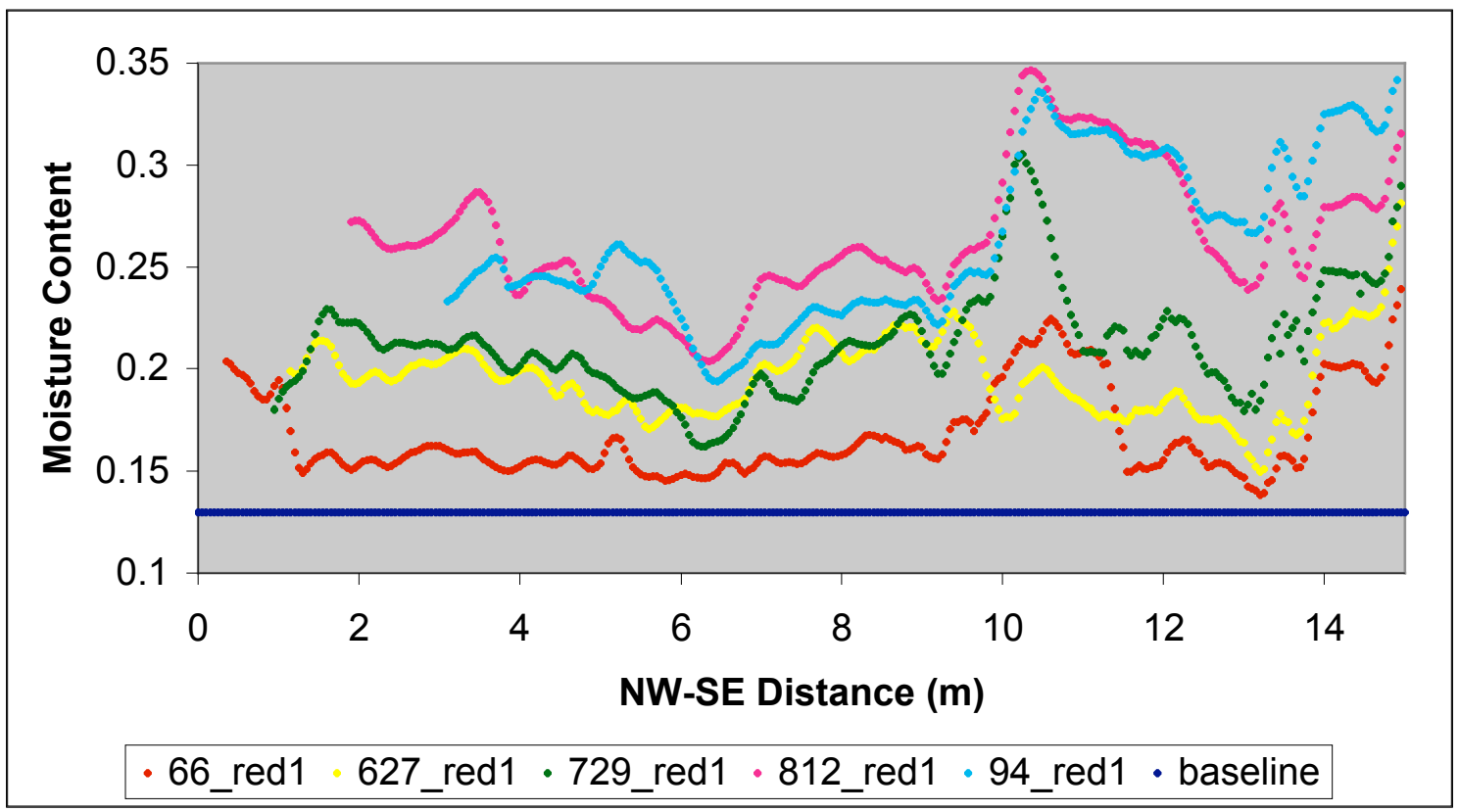

Figure 6: Change in $\square_{v}$ of sediment overlying reflector 'red1' over three-month period.

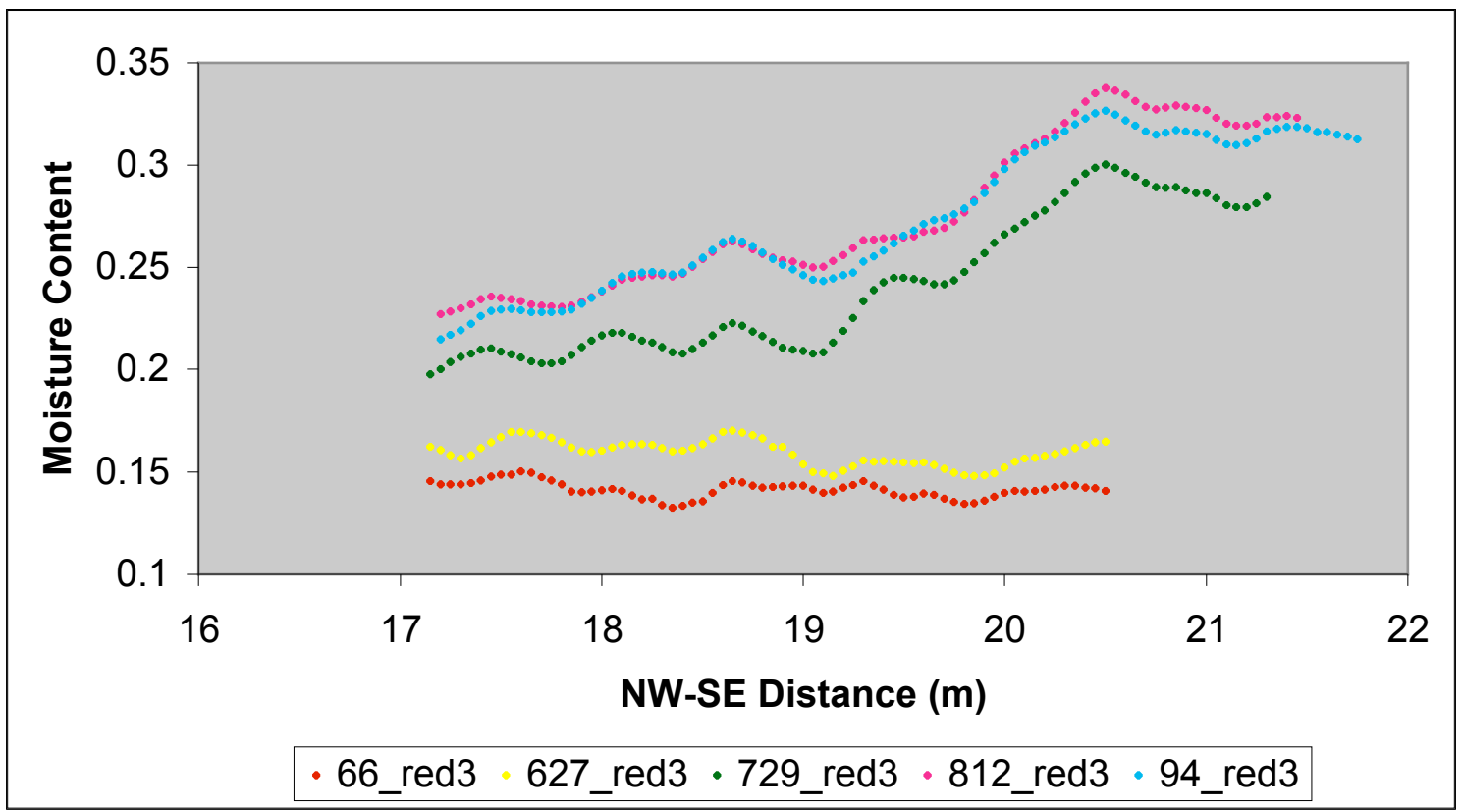

Figure 7: Change in $\square_{v}$ of sediment overlying reflector 'red3' over three-month period. 


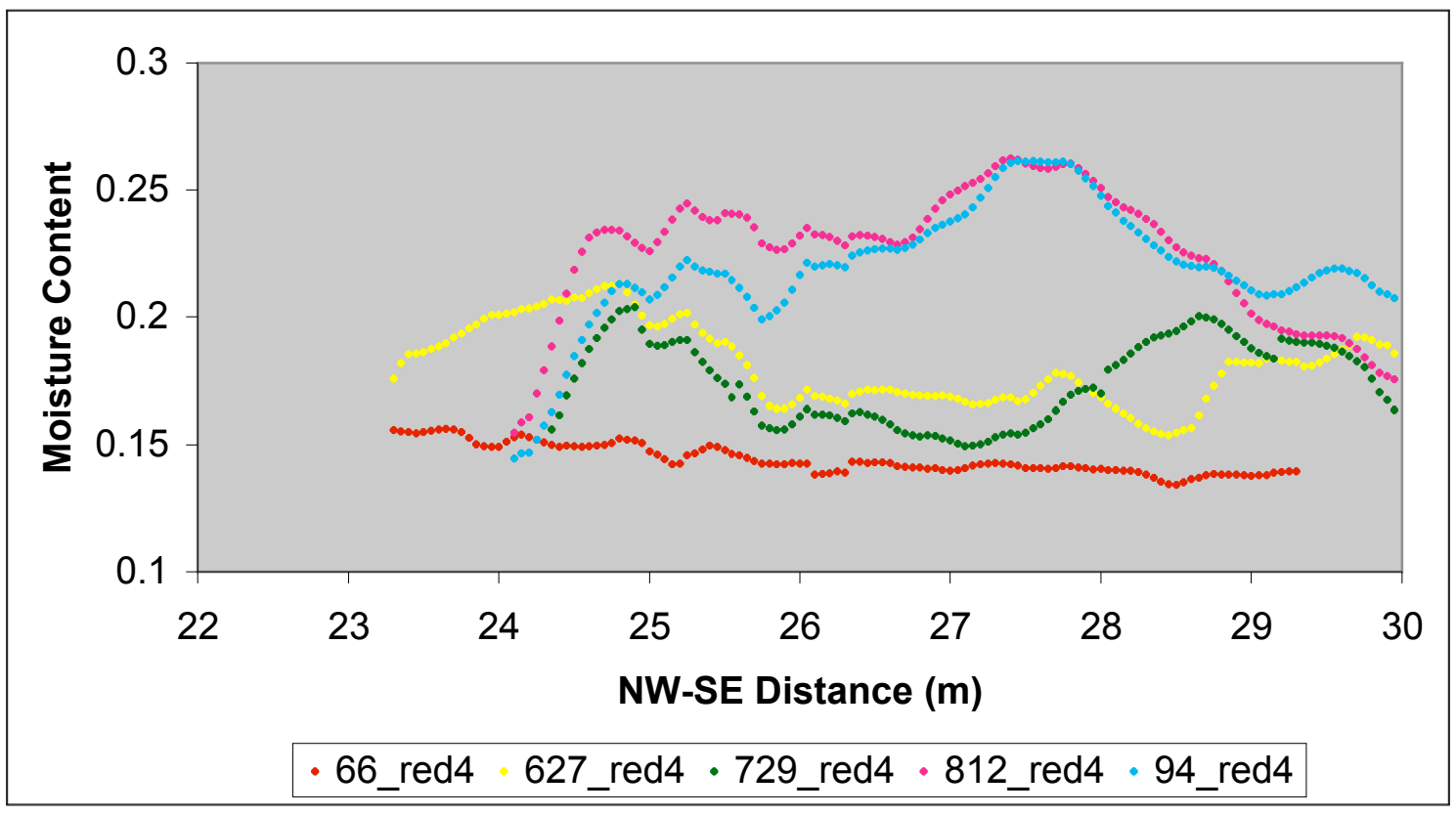

Figure 8: Change in $\square_{v}$ of sediment overlying reflector 'red4' over three-month period.

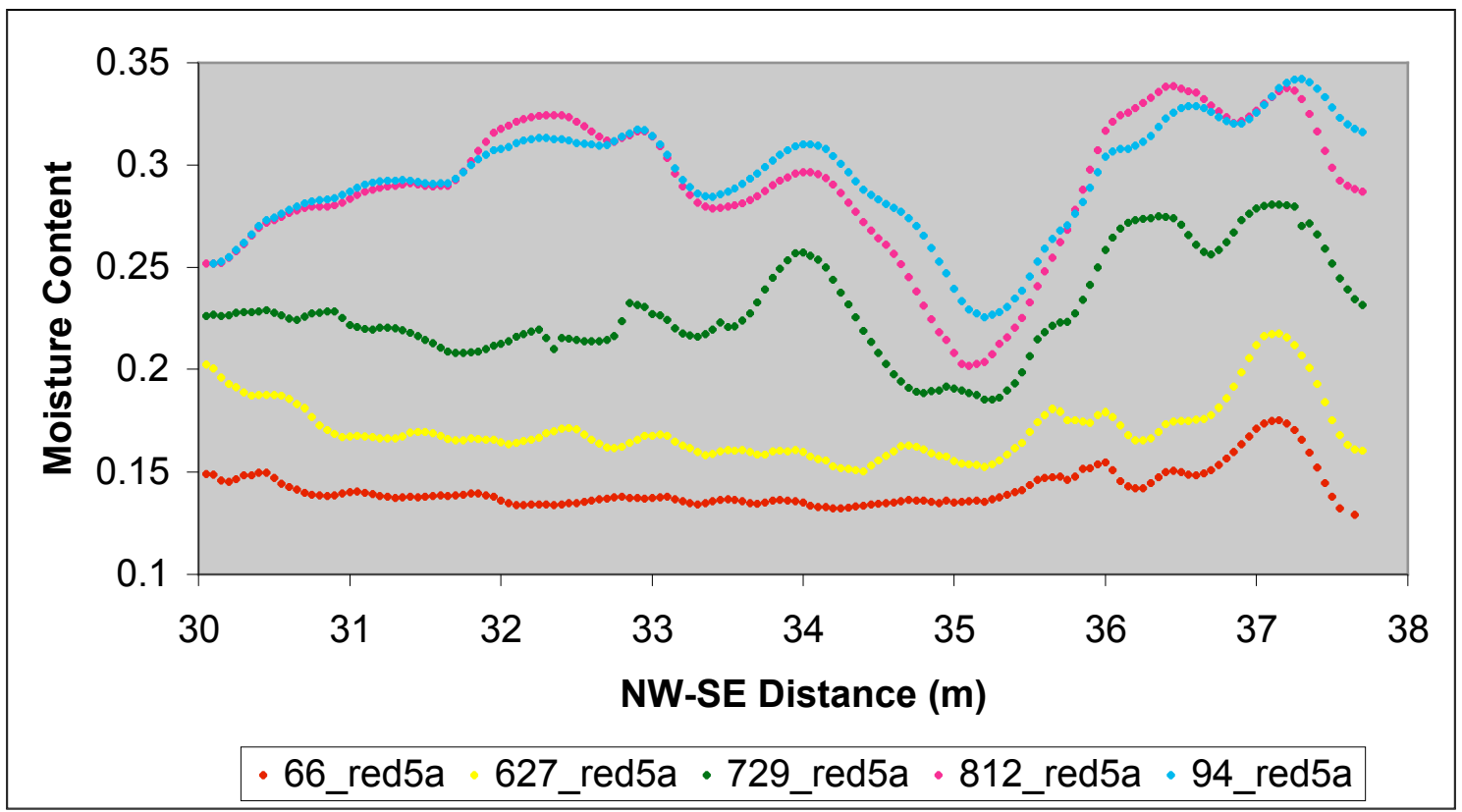

Figure 9: Change in $\square_{v}$ of sediment overlying reflector 'red5a' over three-month period. 


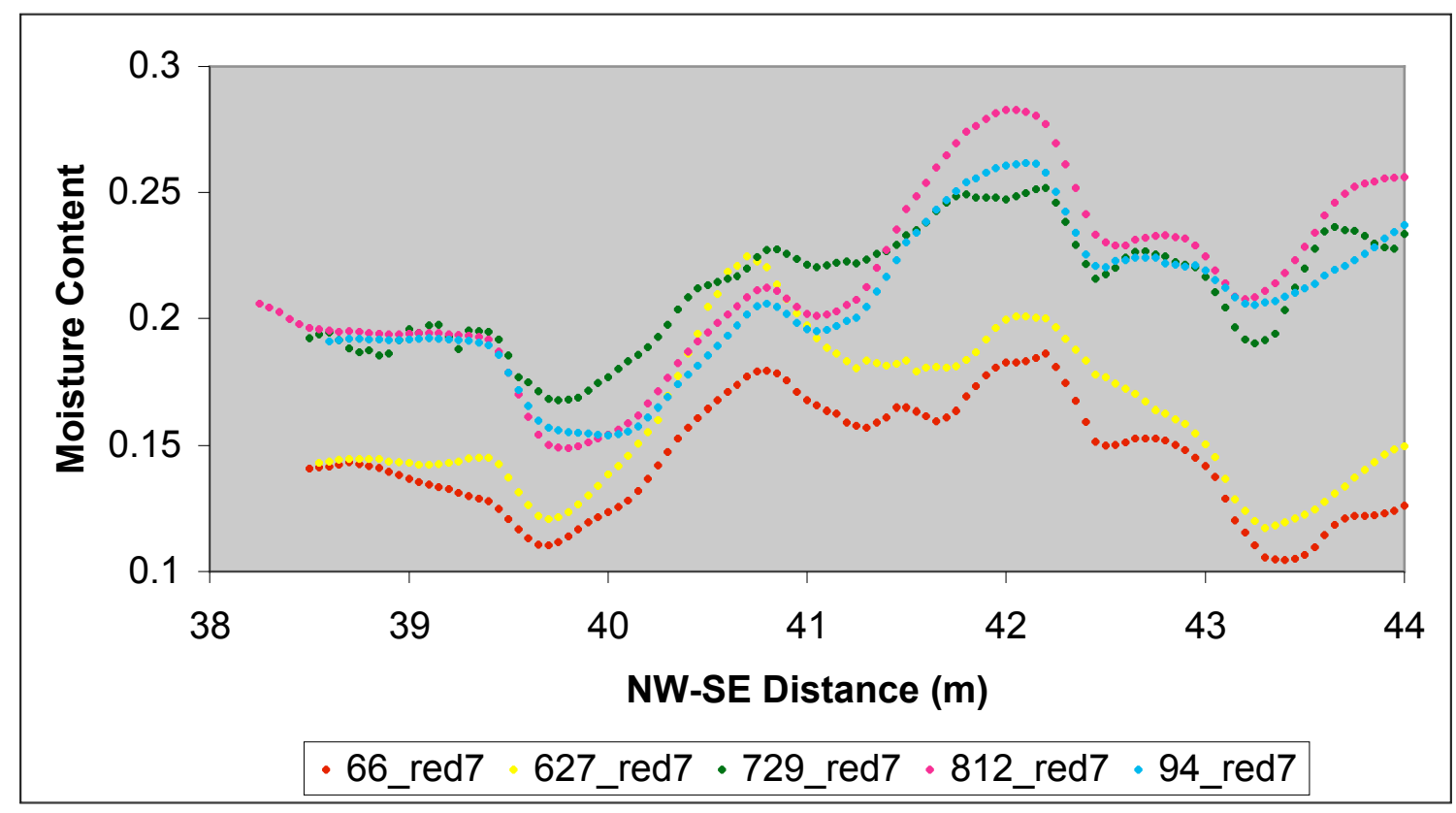

Figure 10: Change in $\square_{v}$ of sediment overlying reflector 'red7' over three-month period.

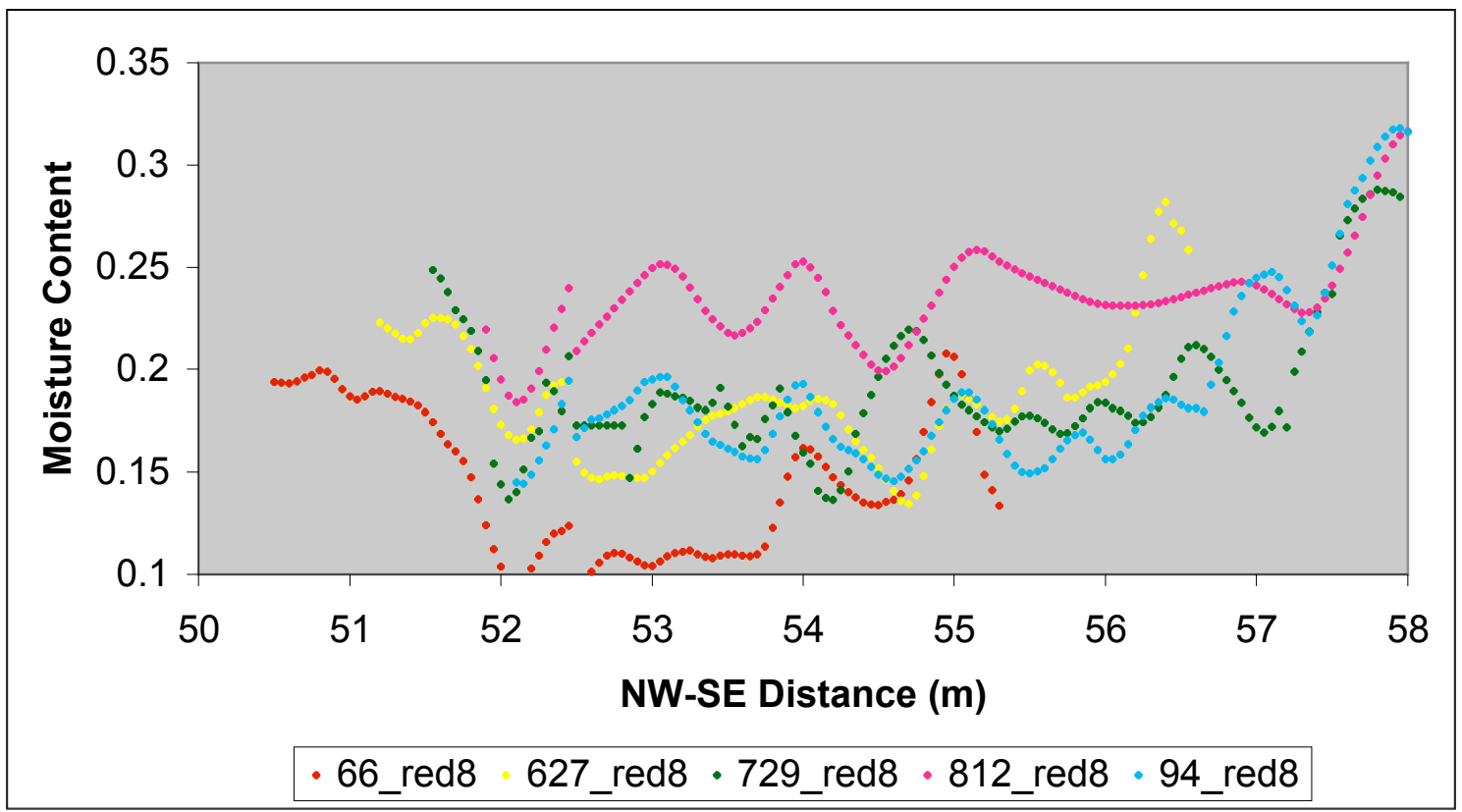

Figure 11: Change in $\square_{v}$ of sediment overlying reflector 'red8' over three-month period. 


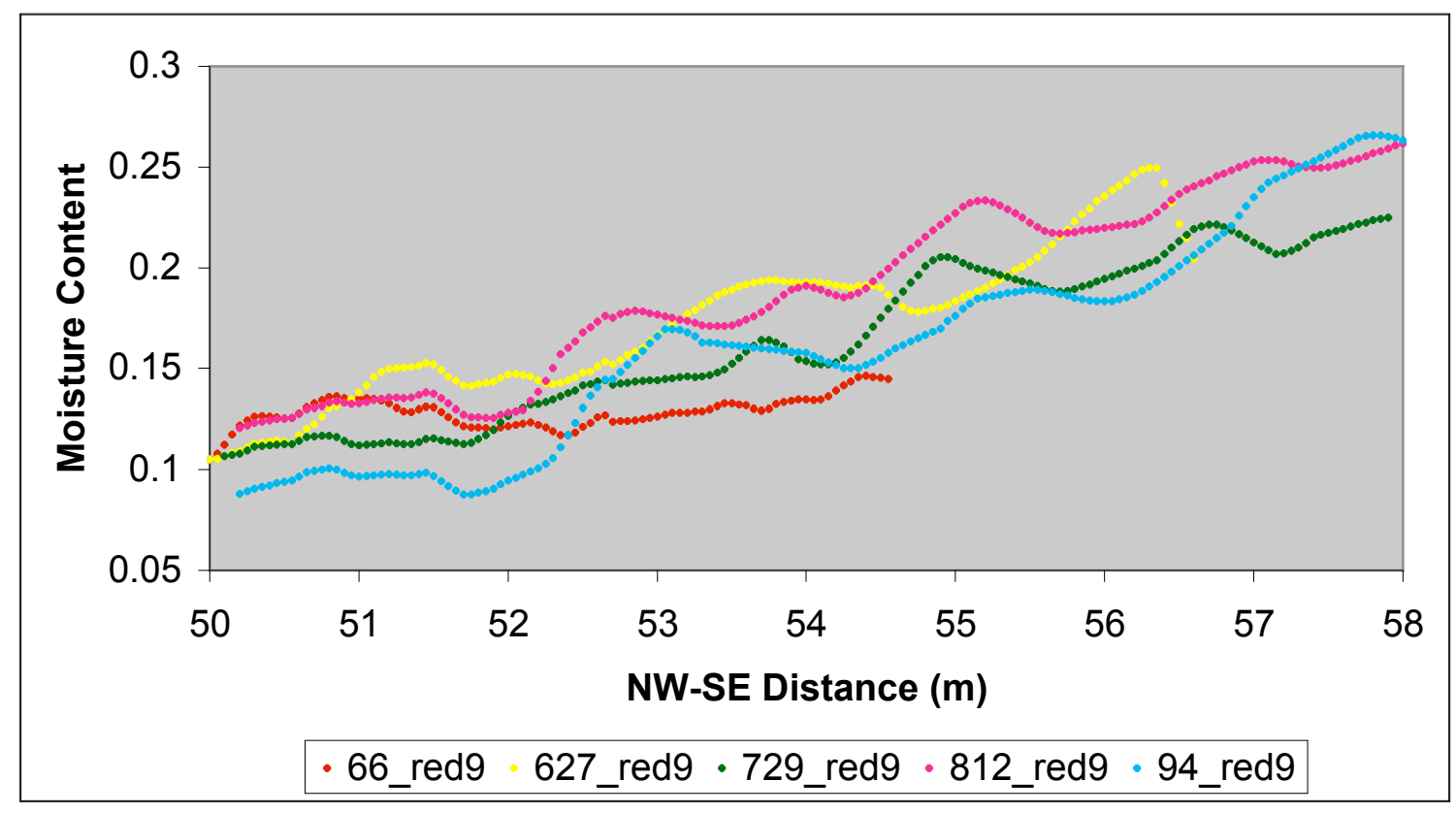

Figure 12: Change in $\square_{v}$ of sediment overlying reflector 'red9' over three-month period.

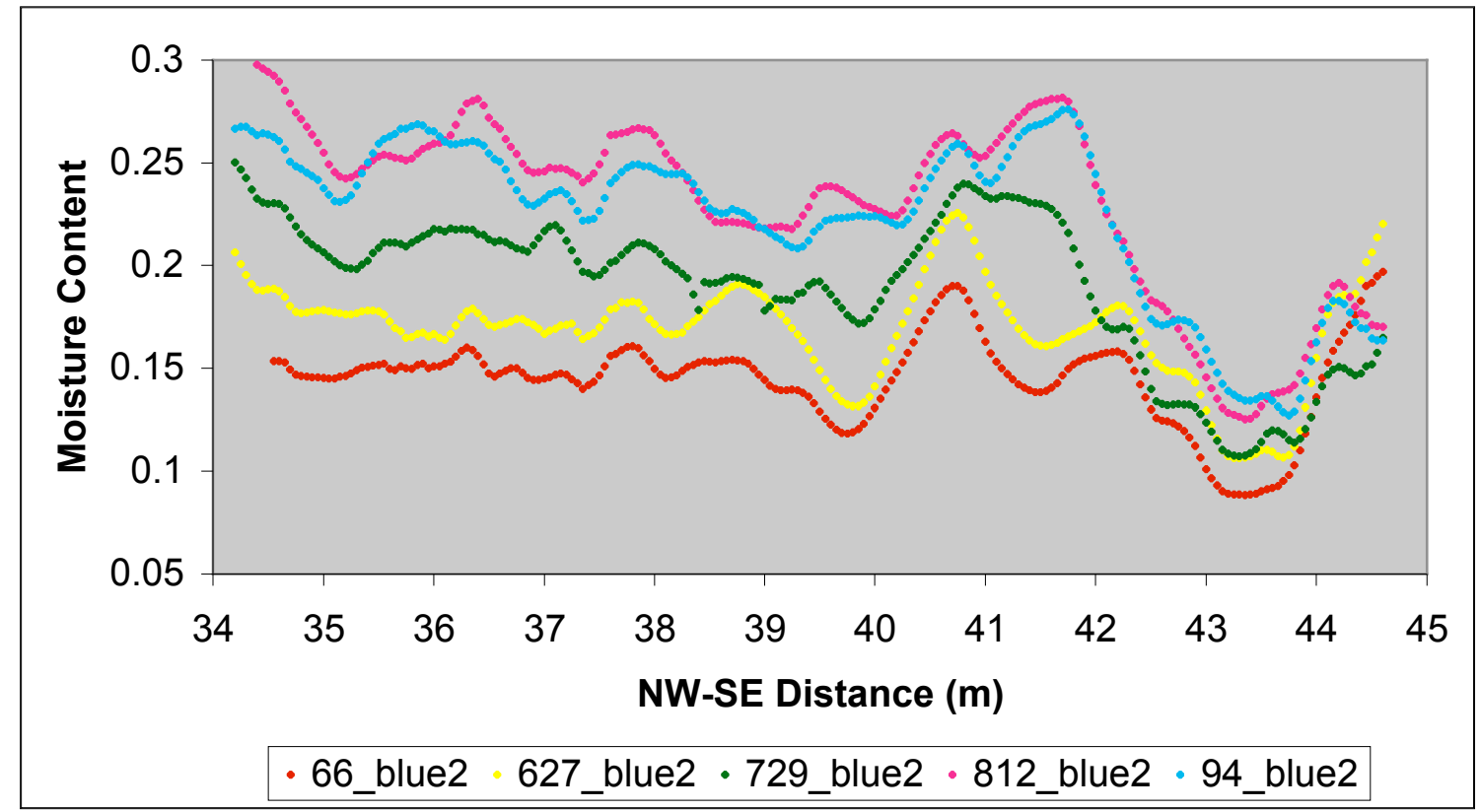

Figure 13: Change in $\square_{v}$ of sediment overlying reflector 'blue2' over three-month period. 
For the most part, each of the plots of $\square_{v}$ as a function of time exhibits the same general trend. As expected, the changes in moisture content were iterative with increases in $\square_{v}$ of approximately 0.05 or five percent per three week interval. Of the eight reflectors, 'red8' and 'red9' were the shallowest. In considering the results for these two reflectors (Figures 11 and 12), it appears likely that the influence of the other near-surface arrivals, such as the direct ground wave contributed to a decreased ability to get good, repeatable travel time picks. This is especially true for the case of 'red8' which, at $0.25-\mathrm{m}$ below ground surface, shows an almost random set of changes in $\square_{v}$ over the course of the investigation. If the data for 'red9' is considered more closely, it's evident that there may be a bit of coherence to the wetting story. The data initially imply wetting over the first weeks of the experiment followed by a cycling of drying then wetting then drying. While this may seem to indicate nothing more than the spurious picking of travel times, the neutron probe data collected in this area (the eastern side of the clastic dike) indicate a similar cyclical change in $\square_{v}$. While an adequate explanation is still elusive, conversations with other project investigators suggest that the influence of a nearby high matric potential sill may be at work acting as a wick to drive moisture migration away from the drip line. It should also be pointed out that the presence of the clastic dike along this portion of the GPR profile might have contributed to the observed anomalous effects. Comparison with the TDR data in this area may ultimately help determine whether the $\square_{v}$ values determined around the clastic dike are of value.

These examples are in clear contrast to the data shown for the remaining reflectors, each of which illustrate quite clearly the progressive wetting of the sediment overlying them. Taking as examples reflectors 'red5a' and 'blue2' (Figures 9 and 13, respectively), one can see the progressive increase in $\square_{v}$ over time - reaching a maximum of nearly 0.35 is some cases. These two reflectors occupy distinct levels within the sedimentary sequence with 'red5a' being on average $0.90-\mathrm{m}$ below ground surface (bgs) and 'blue2' being roughly $0.45-\mathrm{m}$ bgs. As one might anticipate, the time taken for the sediment overlying the deeper reflector ('red5a') to reach a near steady-state value of $\square_{v}$ is longer than for the shallow reflector. The data shown for the other reflectors exhibit a similar pattern reaching steady-state conditions by mid-August (or roughly ten weeks after the 
infiltration started). After this time, the sediment in the near surface (less than 1.0-m bgs) would be at or near field capacity under conditions of gravitational drainage. As more water was released into the sequence, no additional infilling of the pore volume would have occurred and drainage to deeper regions of the sequence would have occurred. The $450 \mathrm{MHz}$ data definitely appear to bear this out. Unfortunately, the limited depth of investigation of these antennas provided little or no information on those depths greater than about one meter. For that, we must turn to the borehole radar data obtained between the boreholes located near the southeastern end of the profile in the region of the clastic dike.

Some mention should also be made regarding the perceived accuracy of the surface reflection methodology, especially as it relates to the fidelity and repeatability of both the acquisition methodology and the picking of travel times. With respect to the acquisition methodology, it became clear early on that the start and stop positions of the profiles were not being exactly repeated. This becomes clear when looking at the $\square_{v}$ data presented above. The prominent peaks and troughs evident in data collected during one visit are phase-shifted from those occurring at later dates. As these peaks and troughs likely represent 'fixed' stratigraphic effects, they would not be expected to change lateral position over the course of the experiment. So the only plausible explanation is the start/stop position of the GPR unit was slightly variable over the course of the investigation and that perhaps some data points were skipped due to a variety causes during operation. This does not diminish the overall utility of the methodology; it merely forces the data to be interpreted a bit more qualitatively. In other words, a given position (e.g. 23.5-m from start) may not correspond precisely each time to the corresponding TDR (or similar) measurement. With respect to the accuracy of the travel time picking, it is helpful to consider the following example. From experience, it appears likely that accuracy of the picks is likely to be on the order of \pm 0.5 -ns. If one then uses this to assess the accuracy of the computed $\square_{v}$ values, then it can be found that the error in $\square_{v}$ is \pm 0.0188 . Smoothing and averaging of the travel time picks probably ensures better accuracy (or at least improved repeatability) of the data and assuming the error is reduced to \pm 0.2 -ns; the corresponding error in $\square_{v}$ is \pm 0.0075 . 


\section{Borehole Radar Results}

\section{Travel-time tomography}

As mentioned previously, MOP and ZOP crosshole surveys were performed prior to infiltration (subsequently referred to as Base), and at three post-infiltration times (subsequently referred to as Post 1 , Post 2, and Post 3, respectively). For the majority of the well pairs $(1-2,2-8,5-4,4-3), 200 \mathrm{MHz}$ antennae were used. However, to obtain a good signal for the well pairs traversing the clastic dike (3-1 and 8-5), lower frequency antennae $(100 \mathrm{MHz})$ were required. After preliminary processing of the raw data (i.e. the zero-time corrections), the travel times were picked, and the two dimensional velocity images were obtained using the crosshole tomography technique described earlier.

Figure 14 shows the base- and post-infiltration velocity tomograms. Several characteristics of the tomograms remain constant throughout the infiltration experiment. Tomograms 5-4 and 4-3 (i.e. the tomograms formed by well pairs 5-4 and 4-3) show relatively uniform velocity up to a depth of $6.0-\mathrm{m}$ at a value of roughly $0.14-\mathrm{m} / \mathrm{ns}$. Tomograms 1-2 and 2-8 show significantly decreased velocity in the upper two meters relative to the lower regions. The tomograms traversing the clastic dike (3-1 and 8-5) show greatly decreased velocities relative to the surrounding tomograms. It is particularly noteworthy that the velocity values do not match up at the boreholes with the neighboring tomograms. This is most likely due to the presence of the clastic dike. In Figure 15, the velocity profiles at the boreholes of the dike-traversing tomographic plane (3-1) are seen to deviate highly from the velocity profiles at the boreholes from adjacent non-dike containing tomographic planes (4-3 and 1-2). This issue is addressed below before discussing any further interpretation of the tomography results in terms of both absolute moisture content and changes in moisture content.

A well-known limitation in crosshole tomography methods lies in their inability to resolve structures that 1) extend vertically above and below the plane of tomography, but 2) have lateral boundaries located between the two vertically oriented boreholes. Simply 
a)

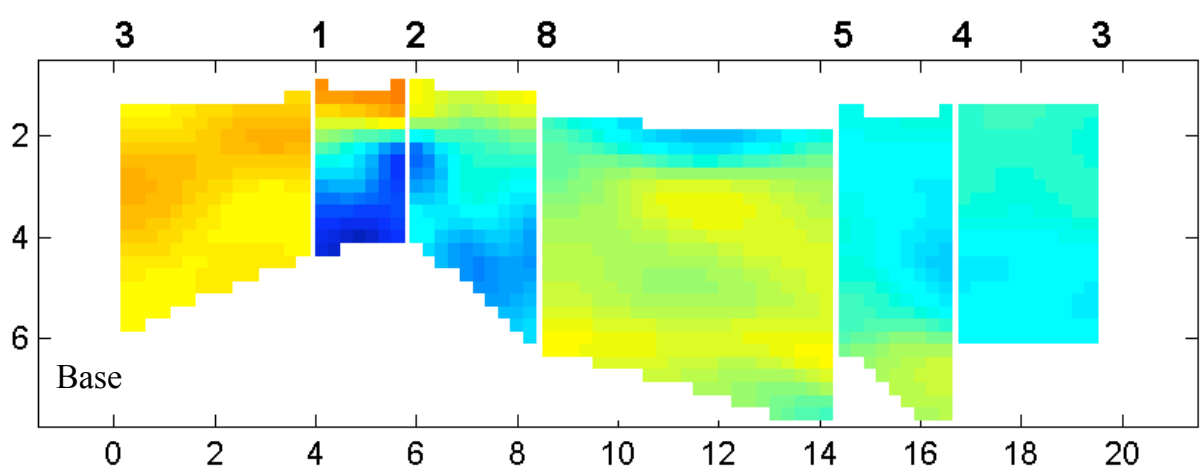

b)

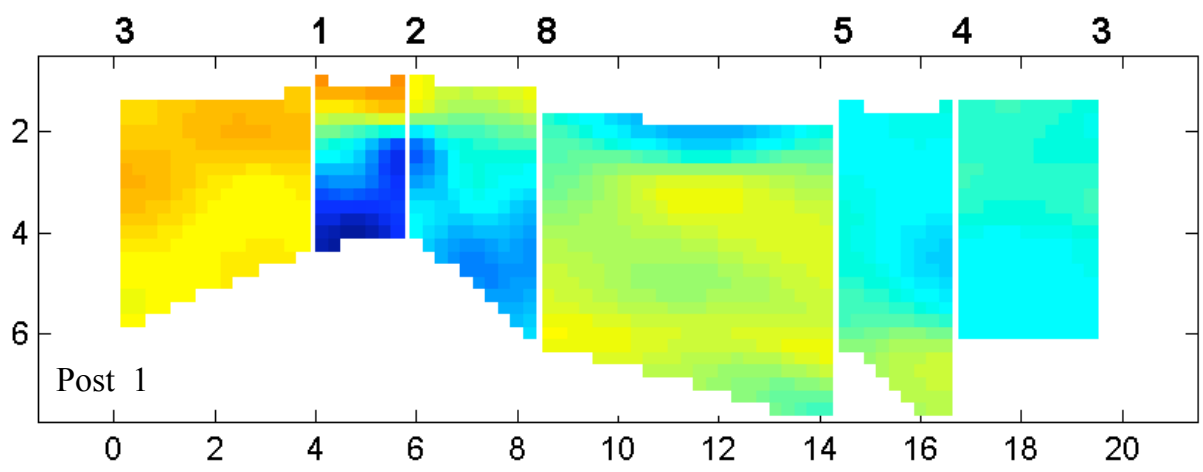

c)

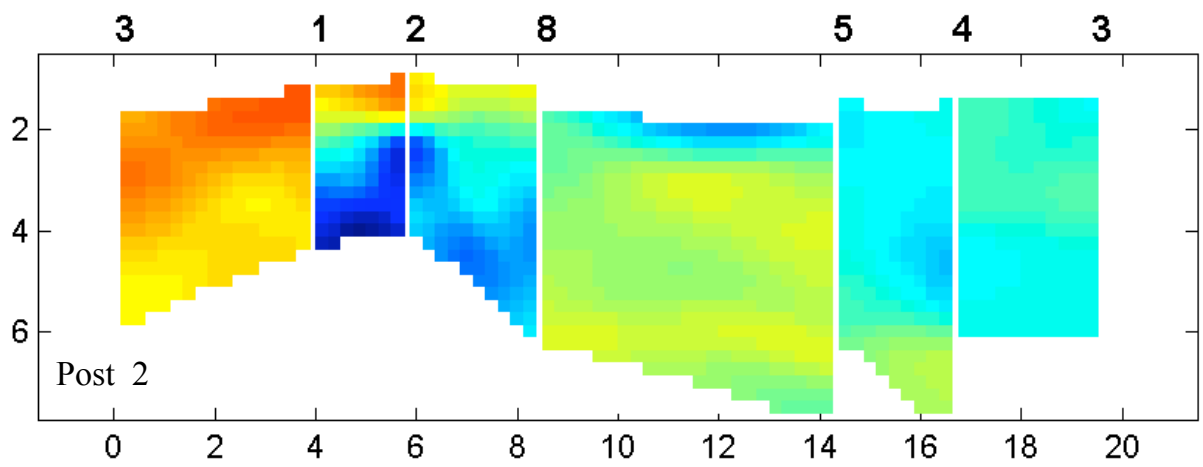

d)

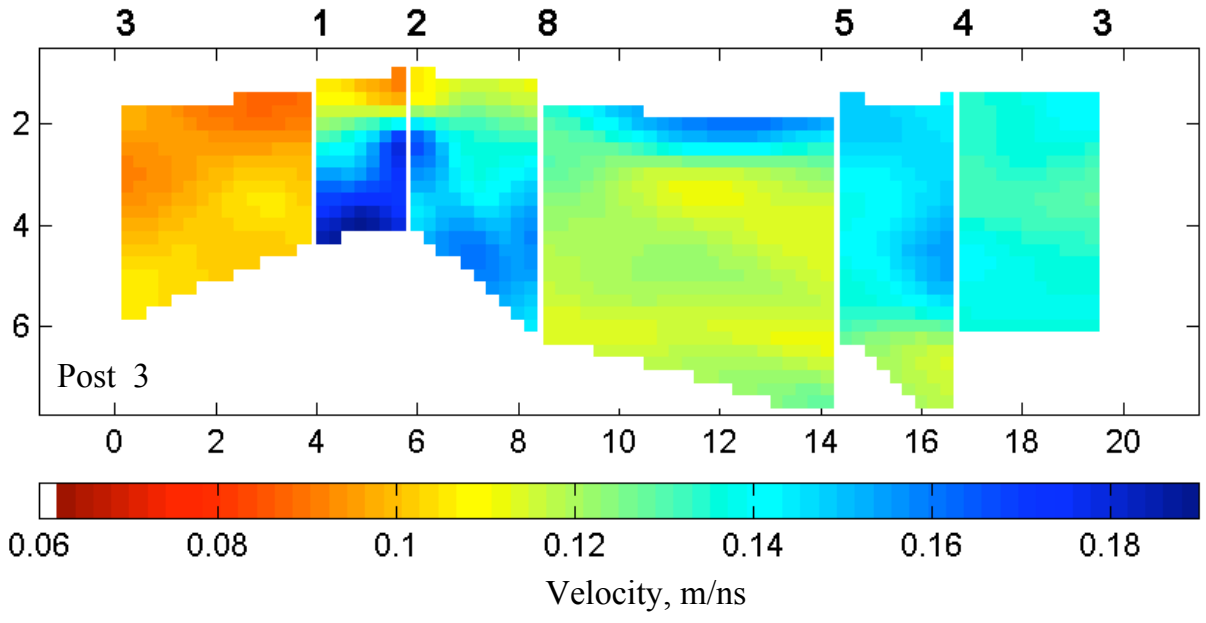

Figure 14. Crosshole Velocity Tomograms (a) before and (b-d) during infiltration experiment. 
put: if no rays can pass around an object (above or below), then the horizontal thickness and the velocity of the structure cannot be estimated from standard crosshole measurement techniques. For the case of a low velocity vertical structure with unknown horizontal thickness and unknown velocity, for example, an overall decrease in velocity is smeared across the velocity tomogram. If, however, the true velocity profile is known on both sides of the vertical structure (say, from neighboring tomograms), and if estimates of the dike thickness are known, then ZOP measurements may be used to estimate the velocity of the vertical structure.

Both planes formed between well pairs 1-3 and 8-5 encompass the clastic dike, as seen through an outcropping at the surface. The dike is presumed to be a vertical structure extending from below the tomogram up to the ground surface, and it is suspected to that contain fine materials with potentially high water retention capacity. The tomograms for these well pairs do not show coherent structure in the vicinity of the dike. Rather, these tomograms show an overall decreased velocity relative to the neighboring tomograms, those that do not intersect the clastic dike. Furthermore, the average velocity value is lower in well pair 1-3 than in well pair 8-5. This is consistent with the fact that the drip line is closer to well pair 1-3 making this region potentially wetter with a lower GPR velocity. These observations can be further quantified by analyzing the relationship between dike thickness and dike velocity with the help of ZOP measurements.

\section{Calculation of dike width versus dike velocity}

Although the clastic dike could not be delineated in the tomograms, an analysis of the ZOP data allowed for a relationship between dike velocity and dike width to be established. If one assumes the width of the dike to be constant throughout the planes of the tomograms, and if one assumes the tomogram velocity values at the wells to be known (from the adjacent tomograms), then the velocity in the dike for a given dike width can be estimated. The procedure is as follows: consider two boreholes located on the left and right sides on a dike of constant thickness. The total travel time $T$ for a 
horizontally traveling GPR wave (such as that collected during a ZOP measurement) is determined by the following relation:

$$
\begin{aligned}
T_{\text {ZOP }}(z) & =T_{L}(z)+T_{D}(z)+T_{R}(z) \\
& =\frac{L \square L_{R} \square L_{D}}{V_{L}(z)}+\frac{L_{D}}{V_{D}}+\frac{L_{R}}{V_{R}(z)} \\
& =\frac{1}{V_{R}(z)} \square \frac{1}{V_{L}(z)} L_{R}+\frac{1}{V_{D}} \square \frac{1}{V_{L}(z)} L_{D}+\frac{L}{V_{L}(z)}
\end{aligned}
$$

where $V_{L}(\mathrm{z})$ and $V_{R}(\mathrm{z})$ are the velocity values to the left and right of the dike, respectively, $L_{L}$ is the distance from the left-most borehole to the left side of the dike, $L_{D}$ is the width of the dike, $L_{R}$ is the distance from the right side of the dike to the right-most borehole, and the total distance between boreholes is known and given by $L=L_{L}+L_{D}+L_{R}$. For a given dike velocity, the unknowns are $L_{D}$ and $L_{R}$.

The above equation can be written in matrix form as $\mathbf{G m}=\mathbf{d}$, where

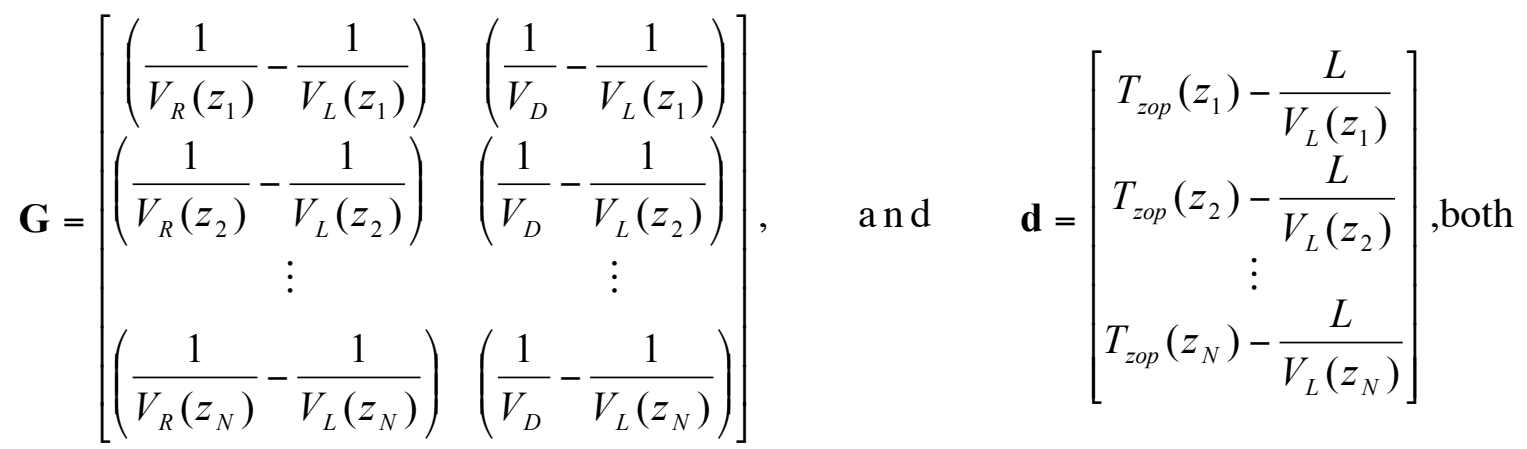

containing only known quantities, $\mathbf{m}=\mathrm{C}_{L_{D}}^{L_{R}}$ is the vector of unknowns, and $\mathrm{N}$ is the number of ZOP measurements.

The least squares solution for $\mathbf{m}$ is given by $\mathbf{m}=\left[\mathbf{G}^{T} \mathbf{G}\right]^{[1} \mathbf{G}^{T} \mathbf{d}$ (Menke, 1989) and it allows for calculation of the dike thickness for a given dike velocity. In other words, the 
dike width value obtained is the value which best produces the observed travel times over the depth of the boreholes given that the velocity distributions surrounding the dike are known from neighboring tomograms.

The various data used for this calculation for the 3-1 tomogram plane are shown in Figure 15. The velocity profiles from the tomogram planes surrounding tomogram $3-1$, and the ZOP-derived velocity profile (also shown) are used to specify $V_{L}, V_{R}$, and $T_{\text {ZOP }}$, respectively.

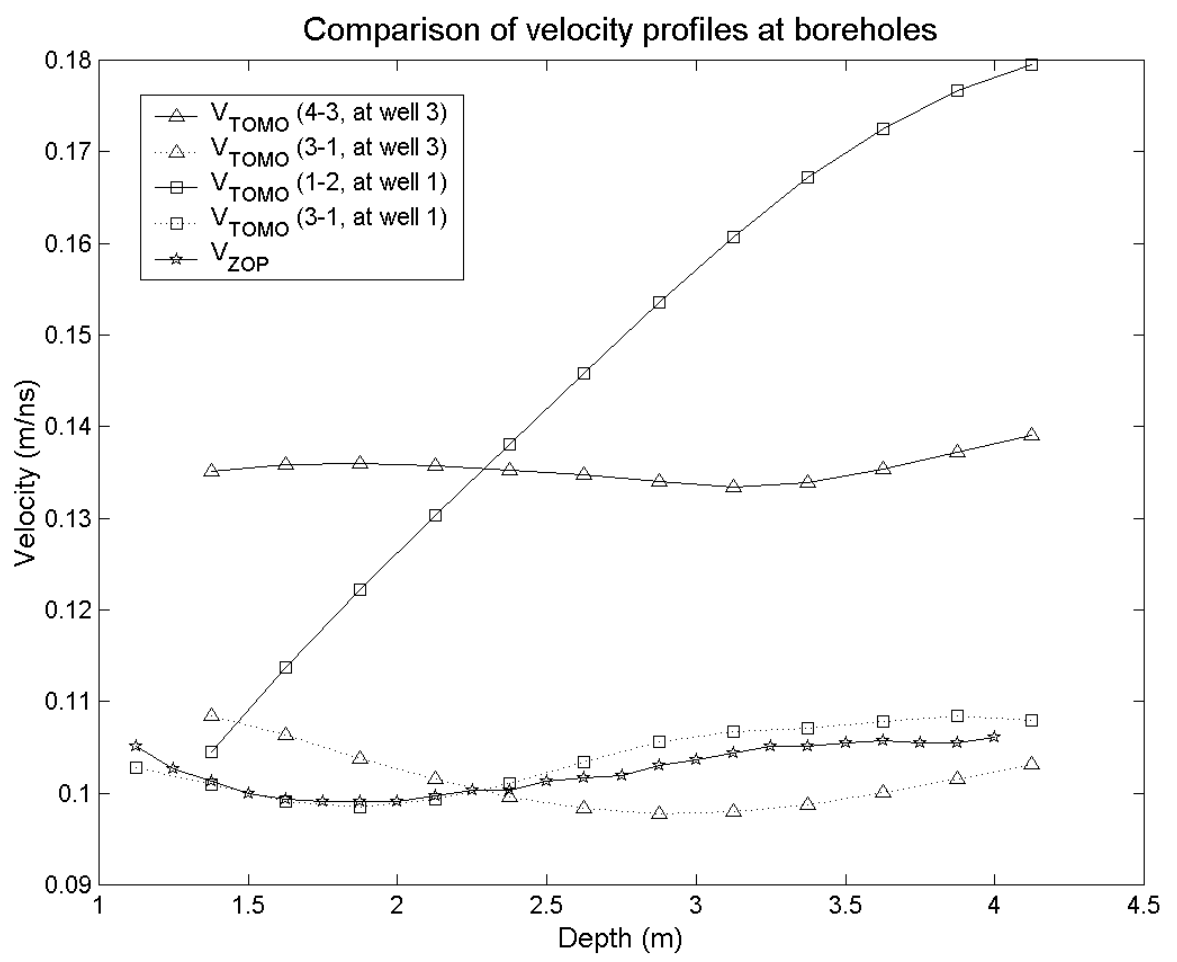

Figure 15. Relationship for dike width versus dike velocity for well pairs 3-1 and 8-5 calculated using ZOP data and velocity profiles from adjacent tomograms.

Figure 16 shows the plot obtained for dike width versus dike velocity for both well pairs 3-1 and 8-5. For a given dike thickness of, say, $1.5 \mathrm{~m}$, the calculated velocity in the dike between wells 3 and 1 is $0.074-\mathrm{m} / \mathrm{ns}$, and between wells 8 and 5 is $0.081-\mathrm{m} / \mathrm{ns}$ (i.e. a 
difference of close to $10 \%$ ). A lower velocity in the dike between wells 1 and 3 could result from higher water saturation in the dike, a reasonable conclusion considering that the drip line is close to this location.

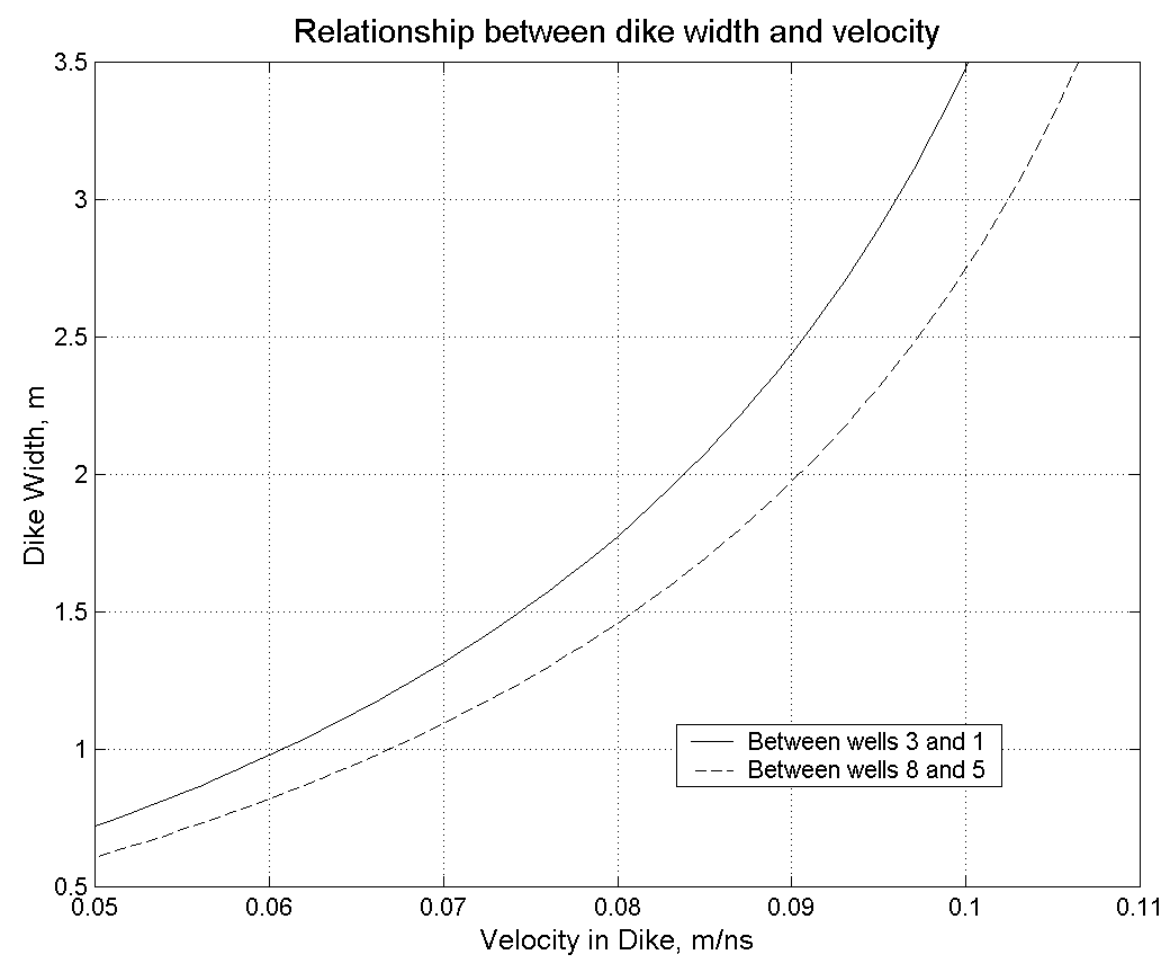

Figure 16. Tomogram velocity profiles at the boreholes of a dike-containing tomographic plane (3-1) compared with velocity profiles at the boreholes from adjacent non-dike containing tomographic planes (4-3 and 1-2). The ZOP-derived velocity profile that is used to calculate the dike thickness versus dike velocity relation is also shown.

Although the tomograms for well pairs 3-1 and 8-5 are affected by the presence of the clastic dike, and therefore, not easily interpretable, it is hopeful that the remaining well pairs may be interpreted in terms of moisture content and changes with time in moisture content during the infiltration test. 


\section{Conversion of velocity tomograms to moisture content}

The tomogram velocity distributions were converted to dielectric constant through the procedure already described. This allows for distributions of moisture content, as shown in Figure 17, to be calculated and compared with neutron probe data (shown in these figures but explained more in depth in the following section). A low velocity region is seen during all times, before and after infiltration, near the surface between well pairs 1-2 and 2-8 suggesting pre-existing elevated moisture content. Regardless, changes in moisture content are observed in this region as infiltration proceeds.

Figure 18 shows the changes from a) Base to Post 1, b) Post 1 to Post 2, c) Post 2 to Post 3, and d) Base to Post 3. Changes in moisture content are relatively constant through much of the region (between $+/-2 \%$ ). Though, there are notable exceptions. For example, the tomogram straddling the clastic dike nearest to the drip line (well pair 1-3) shows significant wetting (dark blue) in the upper region from Post 1 to Post 2--although, as previously mentioned, it is expected that horizontal smearing due to the moist dike (low velocity vertical structure) occurs. Interestingly, slight drying (yellow to red) appears to occur from Post 2 to Post 3 in this same region. Similarly, wetting is also seen in the upper regions of the 1-2 and 2-8 well pair tomograms from Post 1 to Post 2, followed by slight drying in the same regions from Post 2 to Post 3. Significant overall wetting (light blue) is seen from Base to Post 3 in the tomogram corresponding to well pair 4-3. The relevant neutron data are shown for each well alongside the tomograms.

It is important to note that the moisture content range shown is only from $-5 \%$ to $+5 \%$, and through most of the tomograms, changes of only $+/-2 \%$ are seen. Since this is assumed to approach the accuracy limit of crosshole measurements, then the GPRinferred changes in moisture content are assumed minimal. 
a)

b)

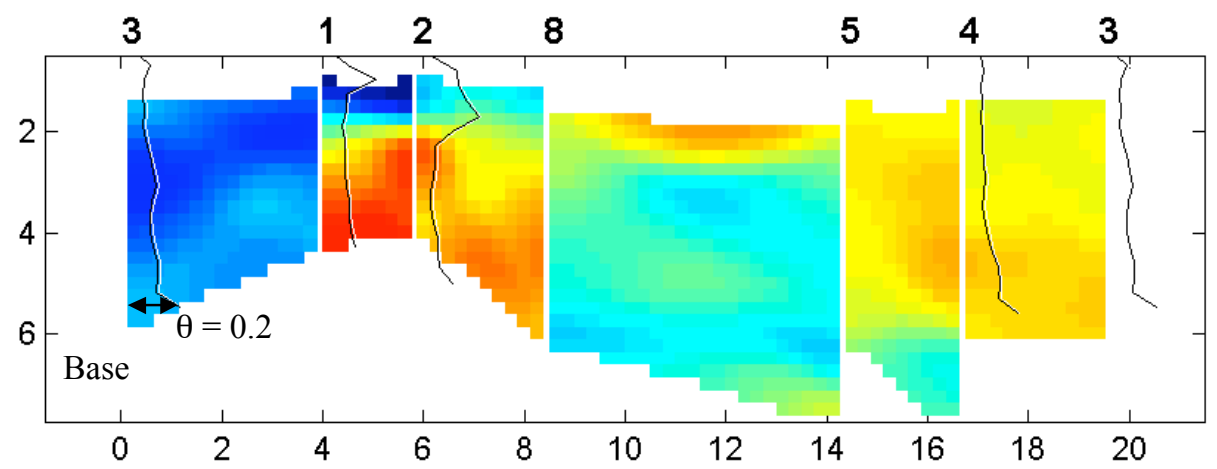

c)
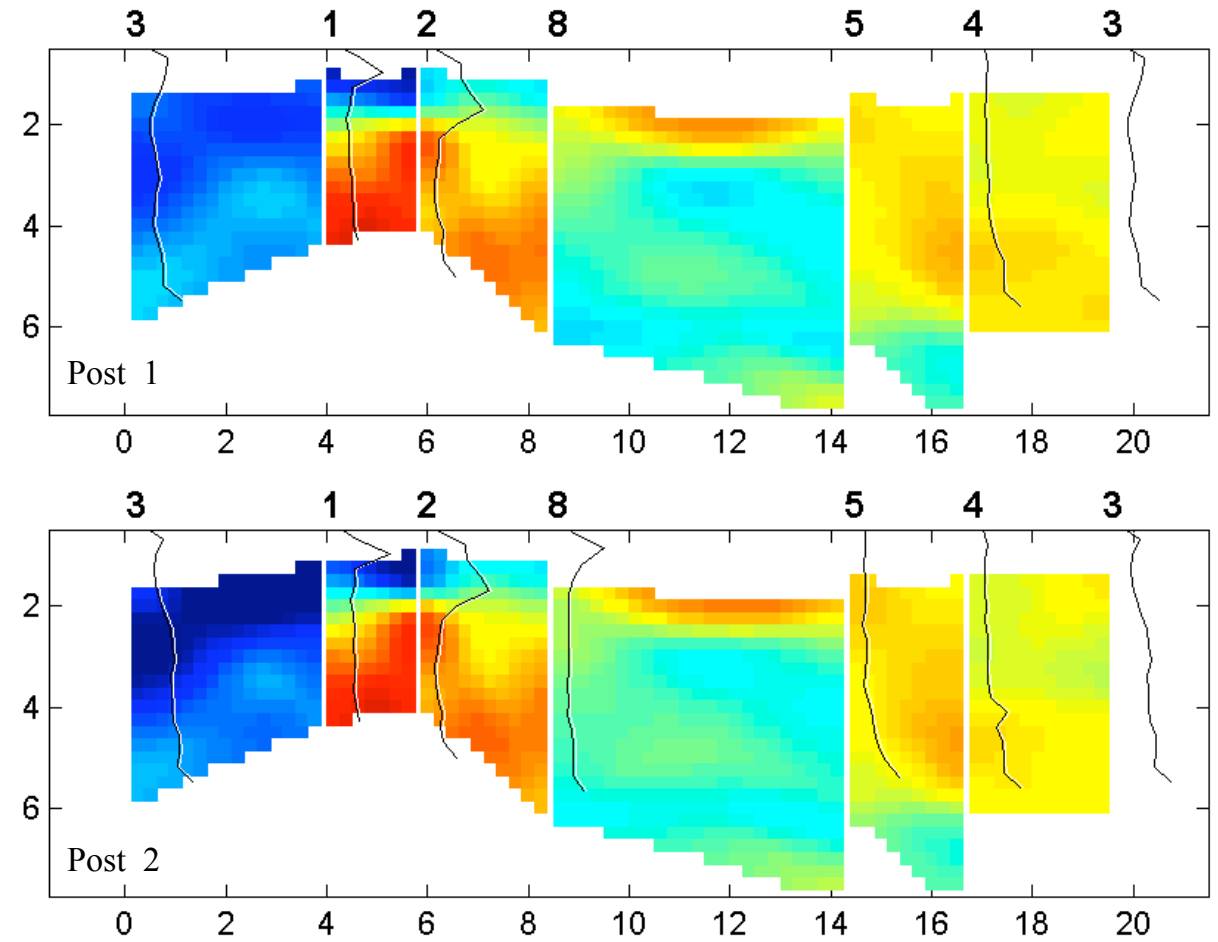

d)

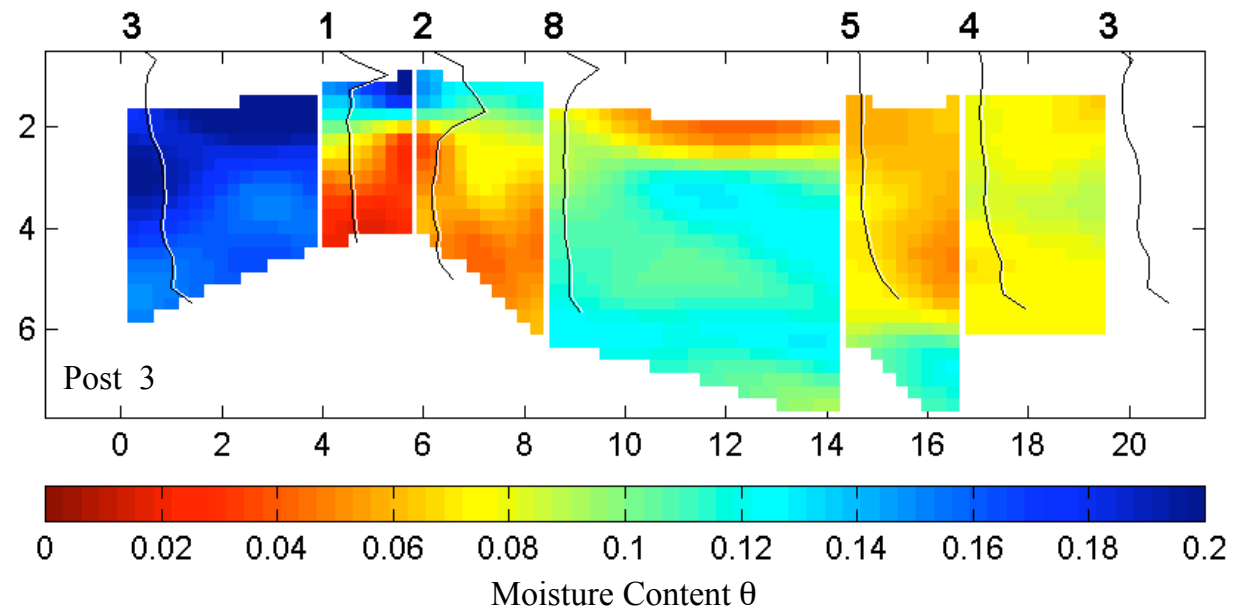

Figure 17. Comparison of moisture content derived from GPR travel-time tomography and neutron-probe measurements 
a)

b)
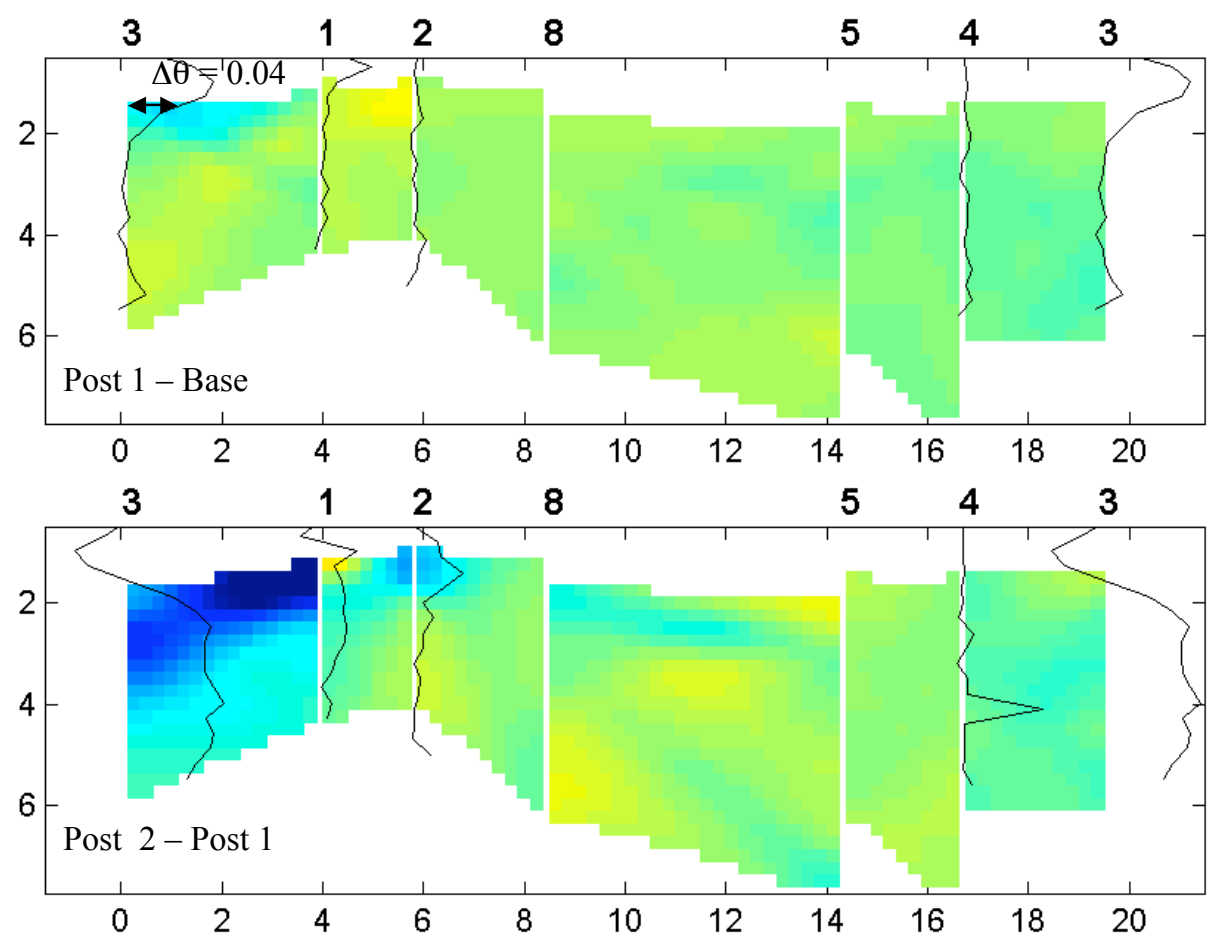

c)

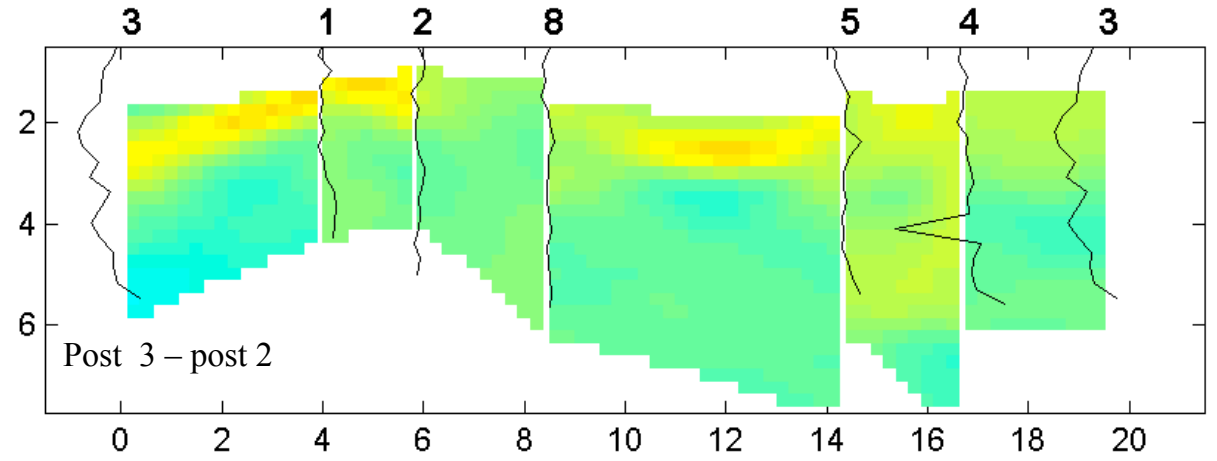

d)
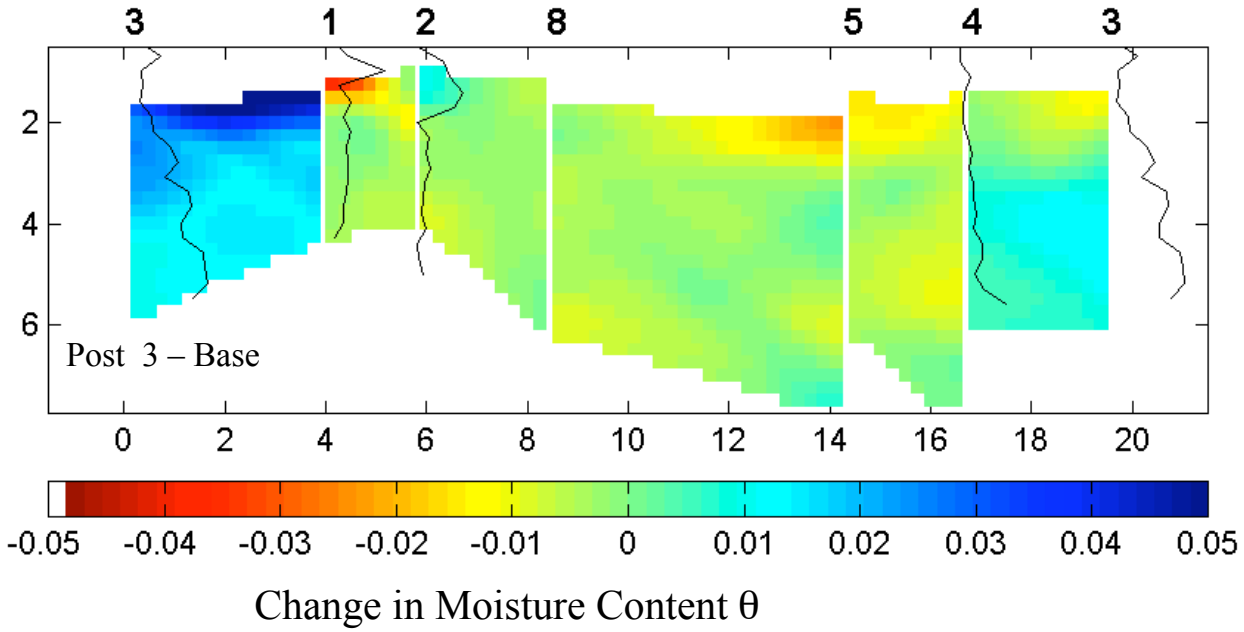

Figure 18. Changes in moisture content derived from GPR travel-time tomography and neutron-probe measurements 


\section{Neutron probe data}

Neutron probe data were also collected for most of the wells at the different GPR survey times; these data were calibrated giving moisture content profiles for the regions surrounding the boreholes. In this way, the neutron probe data may be used to constrain interpretations offered by the GPR crosshole measurements. Figure 19 shows how the moisture content profile (as estimated from neutron probe data) evolves with time. The crosshole tomography surveys correspond to the dates listed in the table (in bold): the Base, Post 1, Post 2, and Post 3 surveys were taken close in time to neutron probe measurement numbers (NP) 2, 3, 8, and 21, respectively. All of the moisture content values are plotted with the same color scale.

Considering wells 3, 4, and 5, one sees that before the onset of infiltration, the moisture content in well 3 is roughly constant (ranging between 0.05 and 0.10 ) above the depth of 4.0- $\mathrm{m}$, below which the moisture content slightly increases. After the onset of infiltration (after NP2, for reference), increases in moisture content to 0.15 and higher are evident, while the region above $2.0-\mathrm{m}$ remains at the lower moisture content $(0.05-0.10)$. Wells 4 and 5 show similar behavior to well 3 before infiltration: nearly constant moisture content values (ranging between 0.05 and 0.1 ) are seen to a depth of about $4.0-\mathrm{m}$, and the moisture content is higher below this depth. These observations suggest that the following flow phenomenon is occurring on the eastern side of the clastic dike: during the infiltration experiment, a predominantly vertically (though somewhat laterally) migrating plume of water developed and reached well 3 at a depth of about 2.0-m but did not reach wells 4 or 5 (probably since they were located further from the water source than was well 3).

Considering wells 1, 2 and 8 (located on the west side of the clastic dike), both wells 1 and 8 show a thin zone of increased moisture content $(>0.2$ : check values) at about $1.0-\mathrm{m}$ depth. Well 2 has a thicker region of increased moisture content between 1.0 and 2.0-m depth. To see how the moisture content varies with time, Figure 20 shows the 

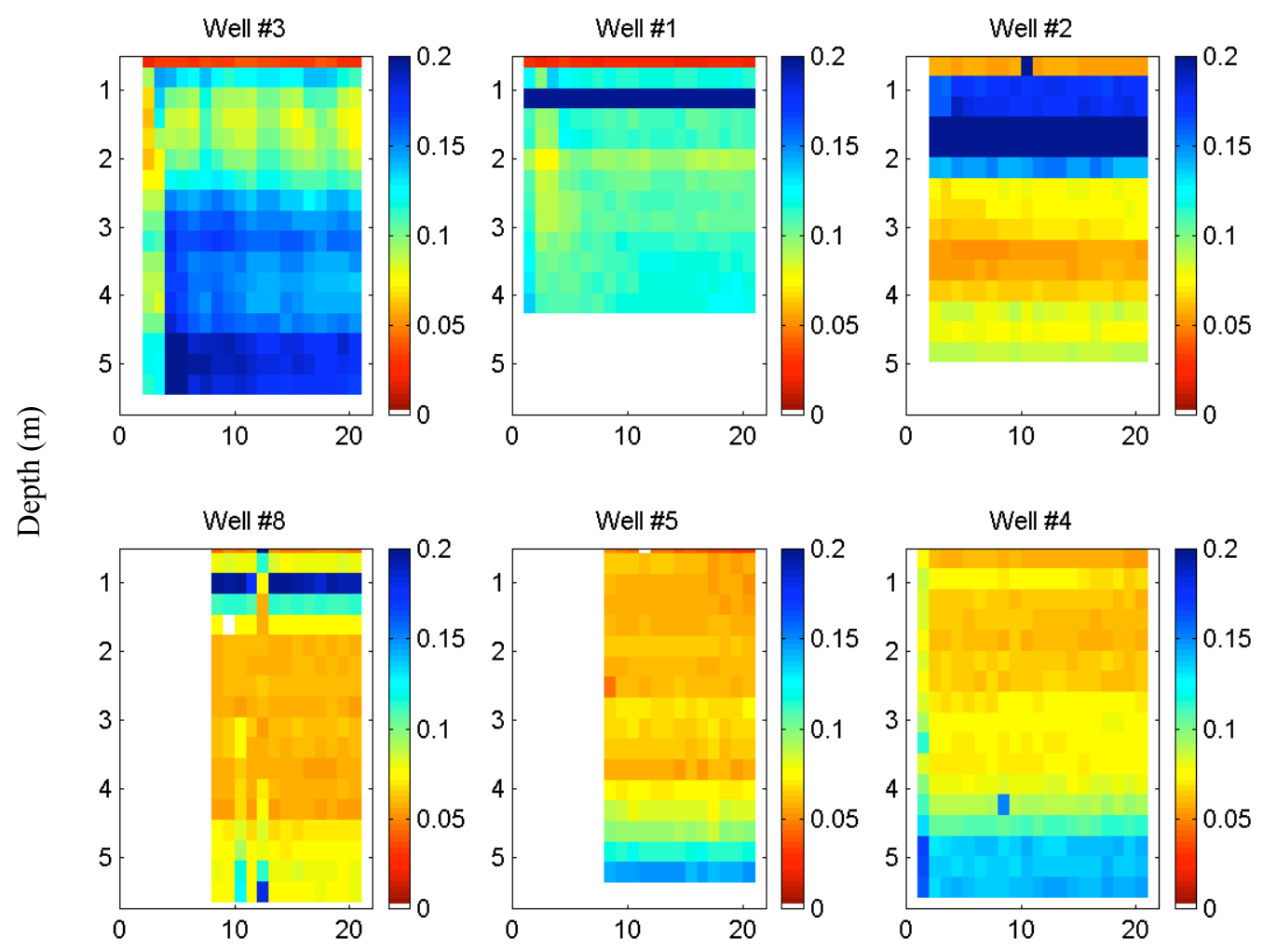

Measurement number, $\mathrm{N}$ (increasing with time)

\section{Measurement numbers (N) and dates}

\begin{tabular}{|c|c|}
\hline 1 & $5 / 30 / 02$ \\
\hline 2 & 5/31/02 (Base) \\
\hline 3 & 6/06/02 (Post 1) \\
\hline 4 & $6 / 10 / 02$ \\
\hline 5 & $6 / 13 / 02$ \\
\hline 6 & $6 / 16 / 02$ \\
\hline 7 & $6 / 20 / 02$ \\
\hline 8 & 6/24/02 (Post 2) \\
\hline 9 & $6 / 28 / 02$ \\
\hline & $7 / 01 / 02$ \\
\hline
\end{tabular}

$117 / 08 / 02$

$127 / 10 / 02$

$137 / 15 / 02$

$147 / 22 / 02$

$157 / 29 / 02$

$168 / 05 / 02$

$178 / 08 / 02$

$188 / 12 / 02$

$198 / 20 / 02$

$208 / 26 / 02$

21 9/04/02 (Post 3)

Figure 19. Neutron-probe-derived moisture content from Base to measurement $\mathrm{N}$ 
difference in moisture content from baseline to later NP measurements. Since baseline NP data were not collected in boreholes 5 and 8, the differential NP values are not available for these boreholes. Again, the most striking observation is the very different mechanism of infiltration observable in both this data and the crosswell radar results. Both methods indicate an almost exclusively vertical migration of fluids to the east of the dike while there is a strong horizontal (i.e. dispersive) component to flow to the west of the dike. This is presumably due to the presence of a fine-grained horizontal sill that - as will be shown below - clearly crosscuts this region and appears to be much wetter than the surrounding sediments.

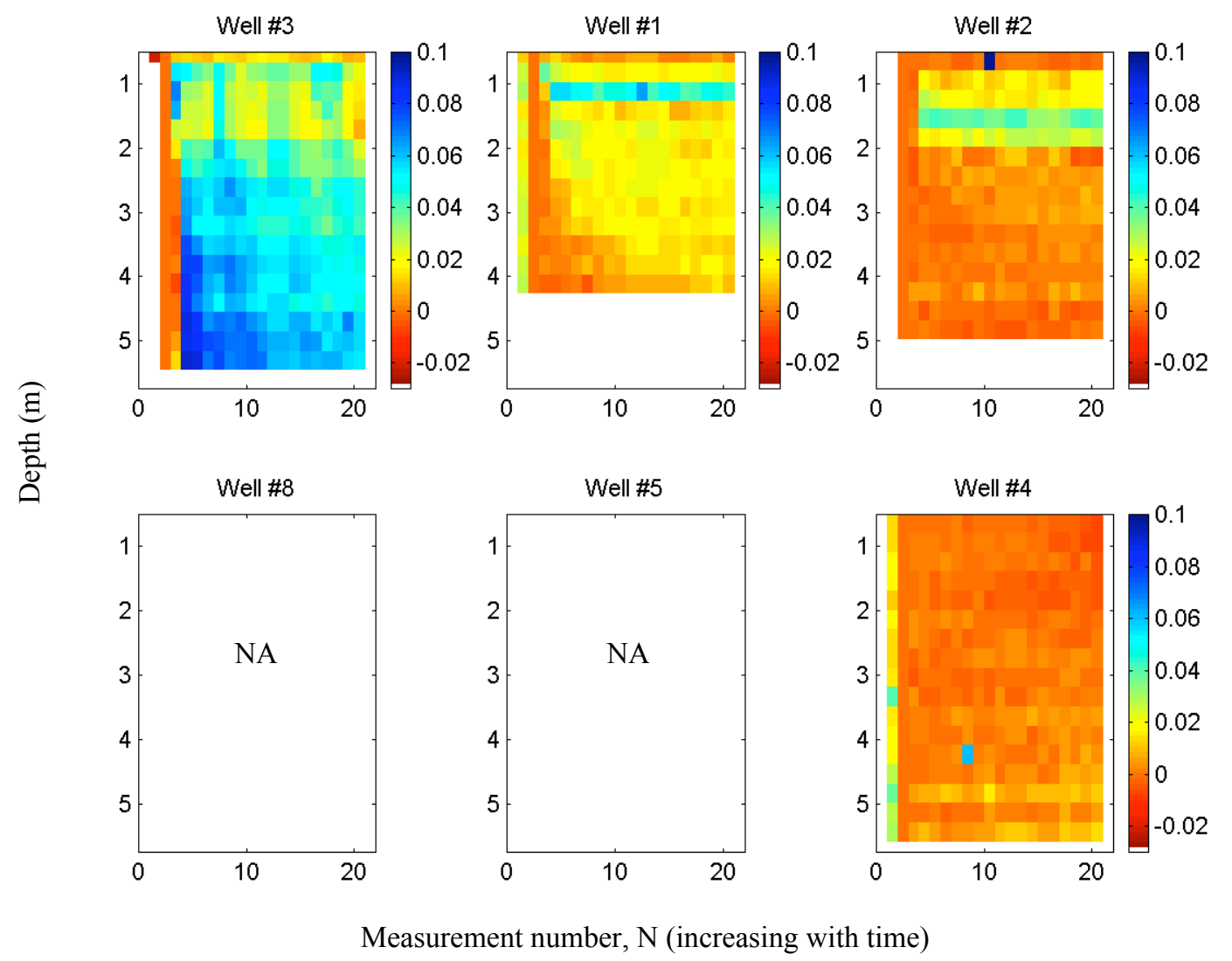

Figure 20. Changes in neutron-probe-derived moisture content from Base to measurement $\mathrm{N}$ 


\section{Attenuation tomography}

The attenuation tomograms also show a similar trend in wetting (Figure 21). Tomograms 1-2 and 2-8 show regions of increased attenuation in the upper two meters. The remaining tomograms are mostly uniform in space. The shallow region of increasing attenuation between 1-2 and 2-8 may be due to a previous injection experiment (FY2001) in this area of the dike in which an electrically conductive tracer (e.g. dyes, thiosulfate, etc.) was used as an injectate. Any partially saturated salts that might have been present at the start of this year's experiment would have acted as a significant attenuator of the radar waves. Previous work in similar sediments has indicated that noteworthy quantities of injected fluids may be retained in fine-grained sedimentary units from one field season to the next. This would provide support for the hypothesis that electrically conductive fluids remained trapped within a similar hydrologic unit (i.e. the sill) in the case of this experiment. Closer examination of the attenuation results indicates that not only are there increases in attenuation over time in the region of tomograms 1-2 and 2-8 but that the initial attenuation values in this region are substantially higher than in other areas surrounding the dike. Furthermore, as new fluids were introduced during the current round of experiments, any pre-existing precipitated salts could easily have been resaturated thereby leading to observed increases in attenuation over time. So it appears that in those regions where changes in differential moisture content are modest due to their initially high degree of saturation (see the neutron probe data results above), attenuation tomography provides a means with which to track infiltration into these regions with a high degree of accuracy. Subsequent infiltration experiments may want to pay close attention to these results and to perhaps consider taking conductive tracer amendments into consideration. 
a)

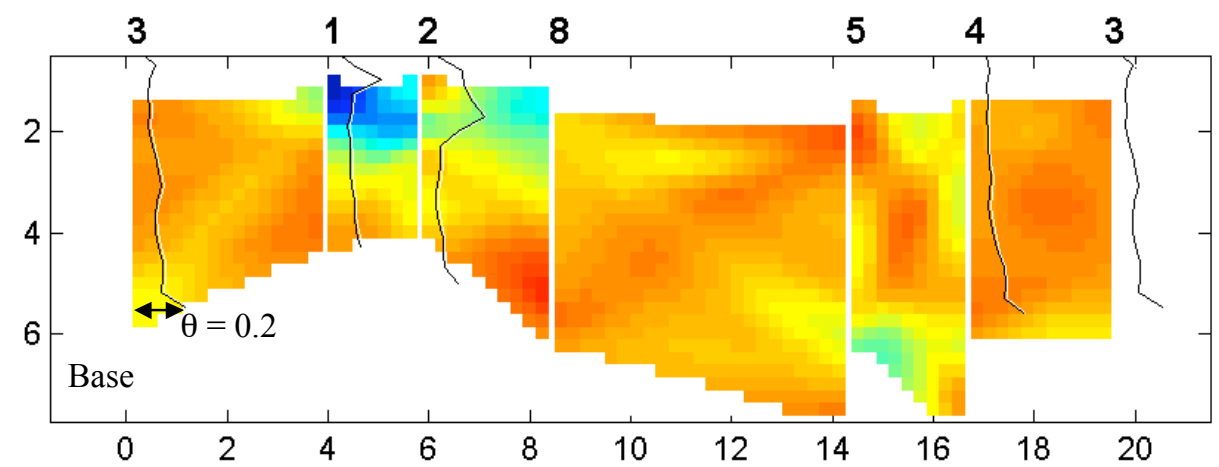

b)

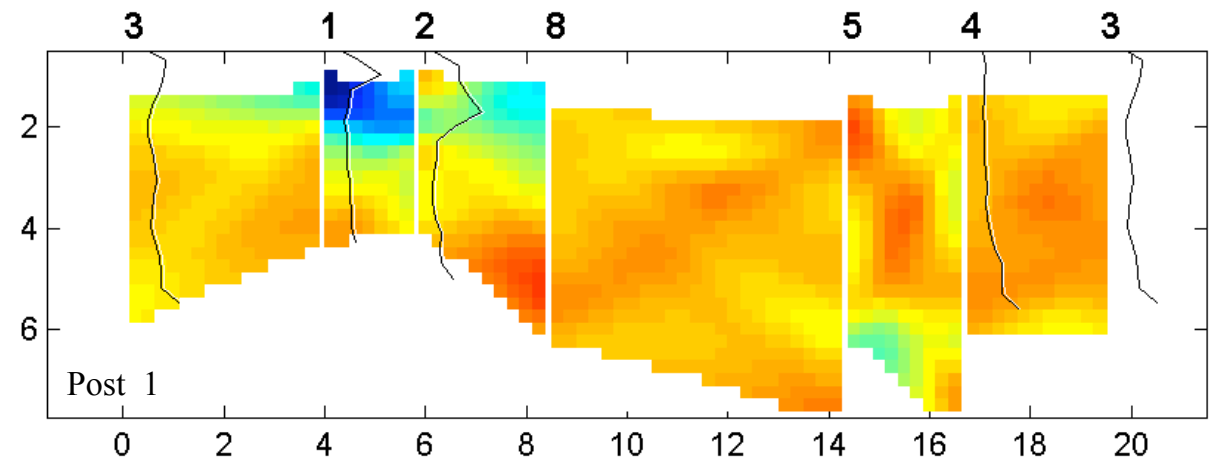

c)

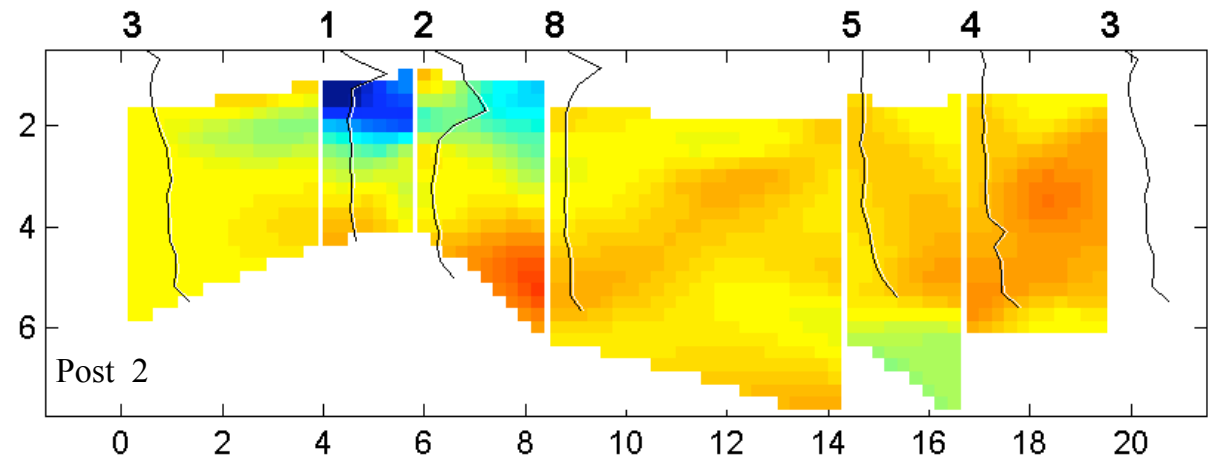

d)

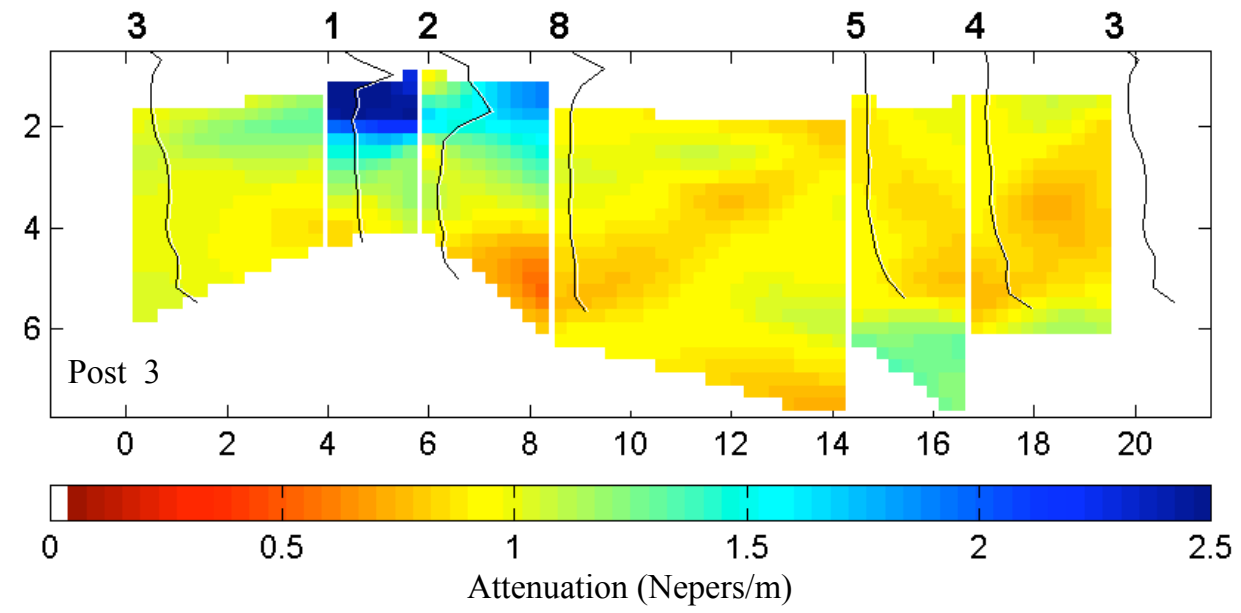

Figure 21. Attenuation tomograms (with neutron-probe-derived moisture content). 


\section{Interpretation and Conclusions}

By integrating the aforementioned datasets, a consistent picture arose regarding the fluid infiltration experiment at the clastic dike vadose zone transport test. The picture that developed was very similar to the one predicted by the initial model governing fluid flow in such an environment - with a few exceptions in and around the clastic dike itself. As predicted, flow fluid (i.e. the wetting front) progressed almost uniformly beneath the drip line with very little horizontal dispersion. The degree of spatial heterogeneity affected fluid flow along the length of the profile, however, is still a bit of a question. Using the results above, one can see several important features that may help answer this question.

When considering the surface GPR measurements, it is clear that there is a strong amount of spatial variability in the degree of wetting. More importantly, the changes in $\square_{v}$ over time determined for each of the reflectors exhibit some degree of spatial dependence. In the case of reflector 'red1,' there are several small $(\square 2.0-\mathrm{m})$ sections of the profile that appear to be preferentially wetting at a greater rate (as evidenced by the rate of $\square_{\mathrm{v}}$ increase) and to a greater degree (as evidenced by the absolute value of $\square_{\mathrm{v}}$ ). A similar effect is observable in any number of the other reflectors but is especially obvious in the data for reflectors 'red5a' and 'red7.' In these cases, not only are there large differences in both the rate of change of $\square_{v}$ and degree of wetting along the lines but also these changes occur over even shorter spatial scales than for 'red1.' The data associated with these two reflectors exhibit change over scales of $\square 1.0-\mathrm{m}$. It should be pointed out, however, that this might be partly (or even largely) an artifact associated with the uniform baseline $\square_{v}$ assumption. Given proper incorporation of the baseline TDR data, one should at a minimum be able to assess whether or not those regions that imply a greater degree of wetting simply started off with greater values of $\square_{v}$.

The conclusions drawn from the surface data are also born out in interpretation of the borehole radar data. The largest changes in $\square_{v}$ reconstructed using the borehole data occurred between the two boreholes (1 and 3) that parallel the drip line. All of the other well pairs located to the north of the drip line exhibited little of the significant wetting 
observed between 1-3. This is not to imply that there were no changes in $\square_{v}$ observed for the other well pairs. Some rather conspicuous changes were noted - all of which were corroborated by the borehole neutron data and all of which appear to be related in some way to the clastic dike and an associated sill. As befits the higher resolution afforded by the borehole radar method, the level of spatial variability indicated by this data was greater than that mentioned above for the surface GPR data. The tomography results indicated that distances separated by $0.5-\mathrm{m}$ or less showed strongly variable changes in $\square_{v}$. Especially encouraging were the observations of similar scales of variability within the trench to the north of the drip line. Clear evidence for lateral dispersion within a thin sill ( $\square 0.25-\mathrm{m}$ thick) was found within the trench that matches a similar region of elevated $\square_{v}$ in the well pairs traversing this region. Evidence for this dispersion - and the fact that it was confined to such a thin layer - is presented in Figure 22, a photograph taken of the exposed sill during the final data acquisition visit.



Figure 22: Photograph of visibly wet sill north of the drip line and west of the dike. 
We have seen in prior investigations at the Hanford site that a combination of geophysical techniques offered another level of detail beyond the traditional hydrologic approaches employed to understand the dynamic nature of fluid flow in the subsurface. The clastic dike vadose zone transport test was no exception. Both the surface and borehole radar techniques offered additional insight into the particular nature of fluid flow in and around the unique geology of a clastic dike. In particular, the borehole measurements provided a range of coverage outside of those measurements made directly along the drip line. Had the trench to the north of the line not existed, visible confirmation of moisture migration along the sill would not have been possible. While the neutron logging was helpful in elucidating this migration when it directly intersected the boreholes, the inter-well coverage provided by the borehole radar technique allowed those regions not sampled by boreholes to be investigated. This ability, along with the rather low infrastructural support needed for the surface radar technique and the information it provides, illustrate the utility of performing such geophysical investigations in support of infiltration experiments such as this one. 


\section{References}

Menke, W., 1989, Geophysical Data Analysis: Discreet Inverse Theory, San Diego, Academic Press, International Geophysics Series, 45, p.40-41.

Peterson, J.E., Paulsson, B.N.P., and McEvilly, T.V., 1985, Application of algebraic reconstruction techniques to crosshole seismic data: Geophysics, 50, 1566-1580.

Raymond, J. R. and E. G. Shdo, 1966, Characterization of subsurface contamination in the SX tank farm, BNWL-CC-701, Pacific Northwest Laboratory, Richland, Washington.

Topp, G.C., J.L. Davis, and A.P. Annan, 1980, Electromagnetic determination of soil water content: Measurements in coaxial transmission lines, Water Resour. Res., 16, 574582. 